\title{
La divine comédie dans les Andes ou les tribulations du mort dans son voyage vers l'au-delà
}

Valérie Robin Azevedo

\section{CpenEdition}

\section{Journals}

Édition électronique

URL : https://journals.openedition.org/jsa/484

DOI : $10.4000 / j s a .484$

ISSN : 1957-7842

Éditeur

Société des américanistes

\section{Édition imprimée}

Date de publication : 5 janvier 2004

Pagination : 143-181

ISSN : 0037-9174

\section{Référence électronique}

Valérie Robin Azevedo, «La divine comédie dans les Andes ou les tribulations du mort dans son voyage vers l'au-delà », Journal de la Société des américanistes [En ligne], 90-1 | 2004, mis en ligne le 05 janvier 2009, consulté le 02 septembre 2022. URL : http://journals.openedition.org/jsa/484 ; DOI

https://doi.org/10.4000/jsa.484 


\title{
LA DIVINE COMÉDIE DANS LES ANDES \\ OU LES TRIBULATIONS DU MORT \\ DANS SON VOYAGE VERS L'AU-DELÀ
}

\author{
Valérie ROBIN AZEVEDO *
}

Le présent article examine des récits eschatologiques individuels recueillis dans deux communautés paysannes quechuaphones de la région andine de Cuzco (Pérou). À partir des représentations de la géographie de l'au-delà, il questionne les interprétations qui évoquent des "survivances préhispaniques », avant de s'intéresser aux formes de récupération et de recomposition de l'imaginaire de la mort par les missionnaires à l'époque coloniale. Cette étape historique fournit une clé pour mieux appréhender la nature particulière du rapport qui unit aujourd'hui les hommes à leurs morts, ainsi qu'il se donne à voir notamment à la Toussaint. Ce rituel ouvre un cycle d'échange entre les vivants et leurs morts, possible après le périple purgatoire de l'âme et l'obtention finale de son salut. Ainsi, le caractère pathogène attribué aux défunts durant les funérailles doit être mis en rapport avec les péchés qu'ils portent au moment du décès. [MOTS CLÉS : Pérou, communautés paysannes, mort, âme, eschatologie, évangélisation coloniale, purgatoire, Toussaint.]

La divina comedia en los Andes o las tribulaciones del alma en su viaje hacia el más-allá. Este artículo examina unos relatos escatológicos individuales recopilados en dos comunidades campesinas quechuahablantes de la región del Cuzco (Perú). Partiendo de una reflexión sobre las representaciones de la geografia del más allá, revisa las interpretaciones que las califican de " supervivencias prehispánicas » para luego interesarse en la manera en que ha sido recuperado y recompuesto el imaginario de la muerte por los misioneros de la época colonial. En este periodo histórico encontramos una clave para entender la forma particular de la relación que vincula, hoy en día, a los hombres con sus difuntos, tal como se puede apreciar en Todos Los Santos. Este ritual abre un ciclo de intercambio entre los vivos y los muertos, posible luego del periplo purgatorio del alma y su salvación final. Asimismo, el carácter patógeno imputado a los difuntos durante los ritos funerarios se debe relacionar en los pecados que cargan en el momento de fallecer. [PALABRAs CLAves : Perú, comunidades campesinas, muerte, alma, escatología, evangelización colonial, purgatorio, Todos Los Santos.]

* Laboratoire d'ethnologie et de sociologie comparative, université Paris X-Nanterre [valrobin@worldonline.fr].

Journal de la Société des Américanistes, 2004, 90-1, pp. 143-181. CSociété des Américanistes. 
The Andean divine comedy or the tribulations of the soul in its voyage to the hereafter: This article analyzes individual eschatological narrations registered in two peasant quechuaspeaking communities of the Cuzco region (Peru). Taking as a starting point the representations of the geography of the hereafter, it retakes the interpretations that categorize them as pre-hispanic survival. Further on, it shows how the imagination about death was transformed by the missionaries in colonial times. This historical moment is important in order to understand the current relationship between live and dead human beings, as it can be seen in All Saint's Day. This ritual opens a cycle of exchange between the living and the dead it is only possible when the latter have already concluded their purgatory voyage and have reached their final salvation. In the same way the pathogenic character of the defunct during the funerals must be related to the sins they are charged with in the moment of decease. [KeY words: Peru, peasant communities, death, soul, eschatology, Purgatory, colonial evangelization, All Saint's Day.]

Comme le soulignait déjà Robert Hertz (1970), dans son texte classique sur les représentations de la mort, le décès est rarement perçu comme un événement ponctuel mais plutôt comme le début d'un long processus de transformation. J'apporterai ici en témoignage des discours tenus sur les morts dans la région andine, au sud du Pérou. Les données proviennent de deux communautés paysannes quechuaphones, situées respectivement dans les provinces de Calca et de Chumbivilcas, du département de Cuzco (Figure 1) ${ }^{1}$. J'examinerai en particulier des récits eschatologiques individuels, ces narrations qui relatent le voyage de l'âme de chaque défunt $\left(\operatorname{alma}^{2}\right)$ et ses tribulations dans l'au-delà, appelé l'« autre vie » (huk vida). Ces récits s'inscrivent dans la prolongation de la séquence finale des rites funéraires. Considérons d'abord l'étape précédant le voyage de l'âme. Les funérailles donnent lieu à une fabrication rituelle du mort, dont la destinée est de renaître dans l'autre vie. Les obsèques se déroulent sur plusieurs jours et constituent un processus de détachement progressif de l'âme, qui doit aboutir à sa sortie du monde des vivants. De multiples offrandes (nourriture, feuilles de coca, alcool) sont faites à son intention pour lui procurer des «forces » (kallpa) durant son voyage. Le surinvestissement rituel qui est déployé vise aussi à neutraliser l'agressivité attribuée au mort récent et à enrayer le danger qu'il incarne alors, son contact étant considéré comme pathogène. Durant la dernière étape du rituel funéraire (l'expulsion définitive de l'âme), la famille fournit au défunt les moyens d'engager seul, en tant qu'être spirituel, son voyage vers l'autre vie. Hormis les offrandes déjà mentionnées, des sacrifices d'animaux psychopompes sont effectués. Ils impliquent fréquemment le chien, généralement celui du défunt, qu'on met à mort par strangulation ${ }^{3}$, afin qu'il suive son maître dans son périple posthume. Le sacrifice peut aussi n'être que symbolique. À Lutto, une reproduction miniature de lama est confectionnée pour transporter les aliments du mort. Les dons de nourriture et le lama sont ensuite brûlés pour faire passer leur essence 


\section{DÉPARTEMENT DE CUZCO}
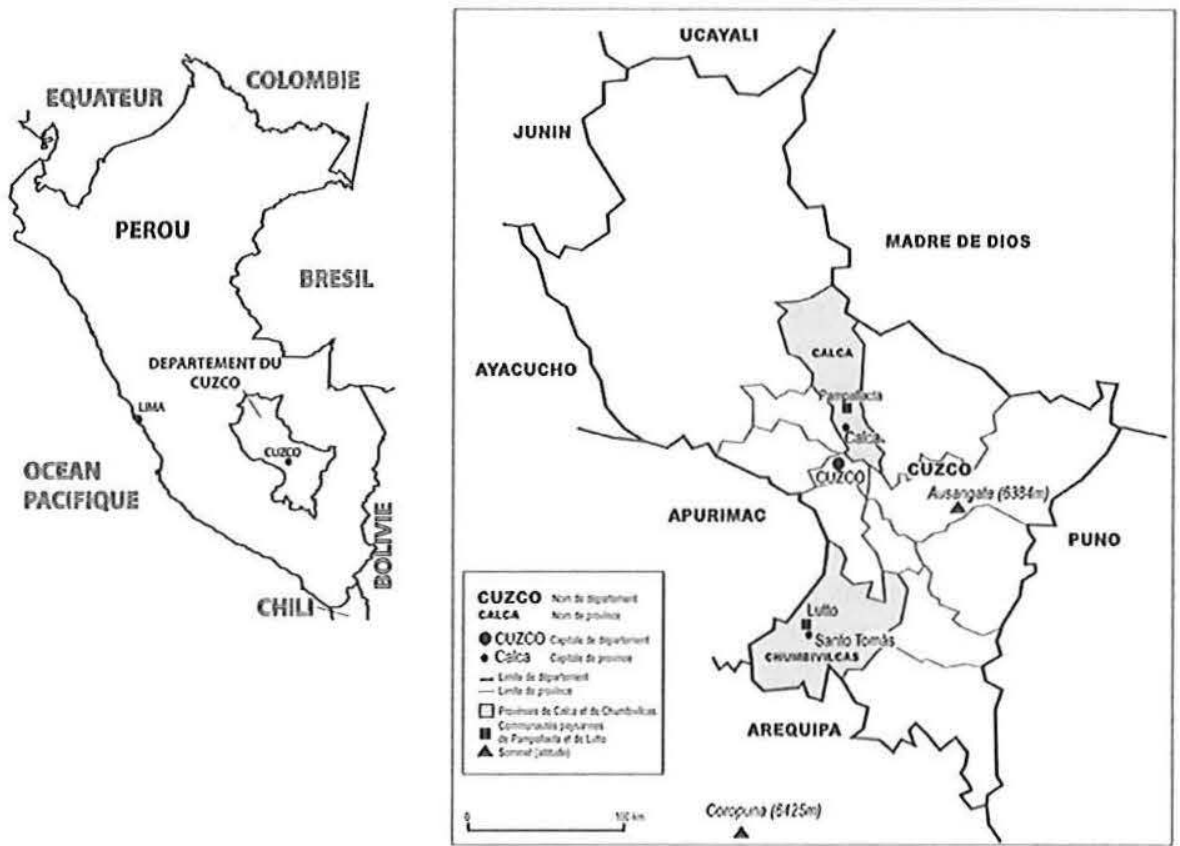

Fig. 1a - Carte du Pérou

Fig. 1b - Carte du département de Cuzco, réalisée par C. et F. Masure (source : J. P. Deler, I. Hurtado, E. Mesclier, M. Puerta, Atlas de la región del Cusco. Dinámicas del espacio en el sur peruano, CBC/IFEA/ORSTOM, Cusco, 1997)

(animu) dans l'autre vie. À Pampallacta, il n'y a pas de représentation figurée de lama. C'est ici le diseur de prières, lequel dirige la dernière veillée funèbre, qui emporte finalement toutes les offrandes destinées au défunt. En fait, à la fin du rituel, il finit par être chassé de la maison des endeuillés, comme une bête de somme. La mise en scène de son expulsion ritualise la sortie de l'âme du monde des vivants ${ }^{4}$. La progression du mort dans l'au-delà est ponctuée par la traversée de différents lieux-étapes (village des chiens, passage d'un fleuve, séjour sur une montagne, généralement un volcan, arrivée au village des morts). L'analyse de ces aventures et de la symbolique des lieux parcourus permettra de mettre en lumière les transformations que subit l'âme pour atteindre le monde des morts (alma llaqta). 


\section{LE DESTIN DE L'ÂME DANS SON VOYAGE POSTHUME}

Après sa sortie du monde des vivants, l'âme emprunte les sentiers de l'autre vie, accompagnée de son chien et de son lama. Pendant ce trajet, le mort, bien que considéré comme immatériel, se comporte comme un vivant et se caractérise par sa corporéité : il cuisine, mange, se repose, dort... La mission du lama dans l'autre vie semble se limiter à la fonction de transporteur d'aliments et autres offrandes. En revanche, le rôle attribué au chien est plus complexe.

\section{Le passage par le village des chiens}

Après plusieurs jours de marche, l'âme parvient au village des chiens (alqu llaqta). Cette localité est une étape cruciale dans l'itinéraire vers l'autre vie et on la retrouve dans la quasi-totalité des versions recensées dans la bibliographie. Si le chien sacrifié lors des funérailles accompagne le mort, c'est aussi et surtout pour l'aider concrètement dans son voyage. Il va d'abord permettre à son maître de franchir ce village où séjournent les âmes des chiens. Il le protège en luttant contre les autres chiens qui attaquent les morts. Mais cette collaboration ne va pas de soi. Cet animal n'aidera pas son maître si, précédemment, celui-ci l'a frappé ou mal nourri. Parmi les chiens présents, le mort peut aussi rencontrer ceux qu'il a élevés au cours de sa vie. Suivant le comportement qu'il a eu à leur égard, ces bêtes, en voyant leur ancien propriétaire, chercheront, ou non, à se venger. Elles pourront, le cas échéant, en faire de la charpie, comme le signale ce commentaire :

Il faut élever un chien avec gentillesse, alors il nous fait passer [le fleuve]. Sinon, là-bas, ils nous arrachent les chairs et nous mettent tout en morceaux. Le chien qui est notre libérateur ne nous fait pas ça. Et, dans le cas où [les autres chiens] nous ont complètement pelés, il nous lèche et nous soigne.

Sumaqta alquta uywakunsi hinaqa chay alqus pasachiwanchis. Mana hinataqsi q'alata chaypiqa peda pedazota peda pedazota aychanchistapas urquyuwanchis. Hinaspas alquqa librasyunninchis mana aqnata ruwawanchischu. Hinaña pelaruwanchis chaypas llaqwaspallas alliyachiwanchis. (Benedicta Huillcayquipa, Santo Tomás, Chumbivilcas, 22/07/2000).

L'âme doit obligatoirement passer par ce lieu, puisqu'il se trouve à proximité du fleuve qu'elle doit franchir pour poursuivre sa route. Elle endure le tourment et survit à cette attaque des chiens dévorateurs. Cet épisode intervertit le rapport de force habituel entre les hommes et les chiens. Mais le phénomène d'inversion avec ce qui se passe sur terre est caractéristique des représentations de l'autre vie. Toutefois, comment comprendre le rôle de ce village des chiens dans le parcours de l'âme?

Le village des chiens est fréquemment appelé « village de Lazare » (Lazaro llaqta) (Loayza 1956 ; Rosell 1976). Nous allons voir que c'est dans son association avec la parabole biblique du mauvais riche et du pauvre Lazare, telle qu'elle 
est traitée dans la tradition régionale, que le séjour dans le village des chiens trouve un éclairage. L’Évangile selon Saint Luc (St Lc 16-19/31) rapporte comment un riche défunt, se retrouvant en enfer, avait eu la vision du pauvre Lazare qui était, lui aussi, décédé et se trouvait désormais dans le sein d'Abraham. Ce patriarche expliqua à l'homme riche, qui avait méprisé Lazare de son vivant, que le sort que chacun avait connu durant sa vie était inversé dans l'au-delà. Rappelons que, dans l'épisode biblique, les chiens ne sont pas absents : ce sont les seules créatures qui s'occupent du pauvre homme et lèchent ses plaies. Par cet acte généreux - le seul dont Lazare bénéficie quand il est en vie -, le chien apparaît sous les traits d'un être charitable, contrairement à l'homme riche. On trouve des traces de la diffusion de cet épisode de l'Évangile dans les Andes aux $\mathrm{XVI}^{\mathrm{c}}$ et XVII ${ }^{\mathrm{e}}$ siècles, notamment entre 1583 et 1649 , période correspondant à la plus forte pression idéologique exercée par l'Église coloniale sur les Indiens (Estenssoro 2003, p. 244). On citera par exemple le Tercero Cathecismo por semones (Troisième Catéchisme par sermons, Tercer Concilio Limense 1867), publié initialement en 1585, à la suite du Troisième Concile de Lima de 1583 qui fixa définitivement le contenu de la doctrine chrétienne, telle qu'elle devait être inculquée aux Indiens. La parabole du pauvre Lazare et du mauvais riche est mentionnée dans le sermon XXX, celui qui devait servir, précisons-le, à l'enseignement du destin posthume de l'âme. De même, cette parabole, qui apportait aux Indiens un espoir de salut (Estenssoro 2003, p. 259), est présente, dans les années 1640 et toujours en lien avec l'au-delà, dans le sermonnaire du curé Francisco de Avila et dans le catéchisme du jésuite Jurado Palomino. De nos jours, ce récit est bien connu dans le département de Cuzco. Sa lecture est courante, tout spécialement lors des services funèbres et des messes célébrées pour les âmes à la Toussaint ${ }^{5}$. Retrouver la figure de Lazare dans les représentations du voyage de l'âme dans l'au-delà n'est donc pas surprenant. D'ailleurs, dans d'autres régions de la chrétienté, la référence à la parabole du pauvre Lazare et du mauvais riche est utilisée pour expliquer le sort subi par certaines âmes au cours de leur périple posthume ${ }^{6}$. Mais il reste à préciser le lien qui existe entre la parabole biblique et le village des chiens andins. Une narration de Julián Poccohuanca, de la communauté de Pampallacta, peut nous aider à donner des précisions. Ce récit fabuleux à propos d'un chien appelé Lazare et de la polémique qui, au paradis, l'opposa à son maître mérite que l'on s'y attarde (voir la transcription intégrale bilingue, en quechua et français, en annexe). Voyons comment son contenu s'inspire de la parabole de l'Évangile selon Saint Luc et permet parallèlement de mieux cerner le rôle du chien dans le parcours du mort vers l'au-delà.

Un riche éleveur de bétail possédait un chien, fidèle gardien de ses troupeaux. L'animal faillit, un jour, à sa tâche et dévora un mouton. Furieux, l'homme le frappa jusqu'à le tuer. Mais le chien ressuscita et s'enfuit. Après avoir traversé un volcan, il trouva refuge auprès de Dieu. Le berger partit à sa recherche et atteignit, 
lui aussi, le paradis où se trouvaient de nombreux autres chiens. L'homme demanda à reprendre son chien et Dieu fit venir l'animal. Mais ce dernier, dont on apprend qu'il s'appelait Lazare - car Dieu l'appela par son nom -, refusa catégoriquement d'aller avec lui et donna ses raisons. Son maître n'avait pas su apprécier son travail et l'avait puni sans commisération pour une seule erreur commise. En représailles de ses mauvais traitements et sur ordre de Dieu, l'homme fut dévoré par un énorme chien avant d'être déféqué par lui. Il dut finalement repartir sans son chien, mais en obtint un autre. Toutefois, grâce aux conseils prodigués à ce dernier par l'animal précédent, le berger ne ramena sur terre qu'un chien fainéant qui passa ses journées à dormir et à manger, sans jamais garder les troupeaux !

On trouve dans cette narration une interprétation libre et originale de la parabole du mauvais riche et du pauvre Lazare. Dans les deux récits, la mort donne lieu à une inversion édifiante du sort des individus. Dans le récit andin, un paysan prend la place du riche. Il est d'ailleurs présenté, dès le début, comme un éleveur qui possède de nombreuses têtes de bétail. Son chien, qui s'appelle Lazare et va au paradis, représente le pauvre Lazare. Ces transformations semblent s'expliquer par la nécessité de rendre le récit exemplaire pour un destinataire paysan, en mettant celui-ci dans la position du riche. Or le seul personnage qui puisse être plus pauvre que le paysan, qui occupe déjà le bas de l'échelle sociale, c'est bien le chien! Dans le récit andin, Lazare ne pouvait être qu'un chien. Le propriétaire a maltraité son chien Lazare de la même manière que le riche, oubliant les paroles des prophètes, est resté insensible et a ignoré le pauvre Lazare, qui vivait à la porte de sa maison, dans l'attente de ses restes de nourriture. Ainsi, à l'instar du riche qui est en proie aux tortures infernales, le paysan propriétaire du chien est puni en se faisant dévorer par un chien beaucoup plus gros que le sien.

Le fait qu'on ait établi un rapprochement entre la situation de l'âme dans le village des chiens et la parabole - en appelant le village Lazaro llaqta-montre bien que le mort doit expier ses fautes dans ce monde inversé qu'est le village des chiens, comme l'homme riche expie les siennes en enfer. En outre, le fait que le chien traverse un volcan avant d'atteindre Dieu, au paradis, situe sans aucun doute possible le récit dans le contexte du voyage vers l'au-delà (nous reviendrons plus loin sur le séjour du mort dans le volcan). Un autre élément confirme cette idée : la scène dans laquelle sont présents de nombreux autres chiens évoque le village des chiens traversé par les morts. L'imbrication, dans le récit de Julián Poccohuanca, entre le passage de l'Évangile et le voyage du mort - dont on a vu qu'ils étaient liés - met en lumière une des fonctions attribuées au chien dans les récits eschatologiques : il incarne le personnage qui châtie le mort en fonction des fautes qu'il a commises. C'est à cause des mauvais traitements infligés au chien par le mort que celui-ci est dévoré. Les épreuves qu'il doit subir font partie d'un chemin pénitentiel qui, nous le verrons, caractérise d'ailleurs l'intégralité du 
voyage de l'âme. Traverser le village des chiens en est la première phase douloureuse. Mais ce n'est que le premier obstacle à surmonter. C'est précisément par la succession d'étapes redoutables que le mort pourra progresser vers l'autre vie. Le passage par le village des chiens agressifs s'inscrit donc dans une vision marquée par la pénitence que doit endurer l'âme pour atteindre sa demeure définitive.

\section{La traversée du fleuve}

Ensuite, le « chien-juge » laisse place au « chien-psychopompe », fournissant son aide au mort pour traverser le fleuve. Le mort va en effet implorer les chiens qui viennent de le malmener afin que l'un d'eux accepte de l'emmener sur l'autre rive en le portant sur son dos. Seul, il serait incapable de le franchir et de lutter contre le courant : il n'a pas assez de force pour effectuer la traversée à la nage ou bien le débit du fleuve, trop puissant, pourrait l'emporter :

Quand nous mourrons, nous partons vers l'autre vie. Notre âme s'en va. C'est pourquoi nous élevons de petits chiens. Nous élevons avec soin un beau petit chien noir comme du charbon et il nous rejoint [dans l'autre vie]. Ensuite il nous fait traverser [le fleuve] à la nage en nous portant avec soin sur son dos. Le fleuve est très puissant et l'eau coule en bouillonnant. Alors le chien nous le fait traverser. C'est ce chien qui nous sauve.

Hinaspa nuqanchis kaymanta wañuspa huk vidaman ripunchis riki. Animunchis ripun. Hinaspa chaymanta alquchata uywakunchis. Chayqa sumaq yana ch'illuchata uywakunchis alquchata. Chayqa ayparamuwansi. Hinaspa ayparamuwaspanchisqa sumaqta q'ipispa nadaspa pasachiwanchis. Supay mayusyá t'impuspa haykushan unu. Hinaspa pasachiwanchis alqucha. Chayqa riki librawanchischá chay alqucha. (Apolinaria Molina, annexe de Ch'alla Ch'alla, communauté de Lutto, Chumbivilcas, 23/07/2000)

Il est remarquable que ce soient toujours des chiens noirs qui aident l'âme à passer le fleuve. C'est la raison pour laquelle on préfère sacrifier un animal de cette couleur lors des funérailles. Contrairement aux chiens noirs, les chiens blancs refusent de faire traverser le mort à la nage, même s'il a été généreux à leur égard durant son existence. C'est que les chiens blancs sont assimilés à la catégorie sociale des mistis ${ }^{7}$. Cette différence entre chiens blancs et noirs reflète la dichotomie entre paysans des communautés et habitants des villages; elle reproduit la relation hiérarchique qui caractérise l'histoire des rapports entre ces deux groupes sociaux. Par ces chiens psychopompes, la position sociale supérieure occupée par le misti dans la configuration régionale est transposée dans la description de l'imaginaire de l'autre vie. L'âme ne doit attendre aucune solidarité, ni aide, de la part des chiens blancs, pas plus que les paysans n'en doivent attendre de la part des mistis. Ainsi, le rôle du serviteur est nécessairement joué par le paysan. L'idée que ce rôle puisse être assumé par le misti apparaît comme saugrenue. La tâche laborieuse du transport de l'âme ne peut donc être effectuée par les chiens blancs. 
Ceux-ci se revendiquent en effet comme étant des «mistis, des messieurs et des dames qui ne portent pas [l'âme] » ${ }^{8}$, ou qui répondent à l'âme : « je suis un misti, je pourrais salir ma chemise, je ne peux pas te porter $"{ }^{9}$. La nature de ces chiens blancs est incompatible avec la réalisation de ce travail pénible et sale - le fleuve étant très impétueux, sa traversée est diflicile ; d'ailleurs, un des noms qu'on lui donne est Map'amayu qui signifie littéralement « fleuve impur ». Il incombe donc aux chiens noirs, représentant les paysans, de traverser le fleuve avec l'âme. On trouve aussi l'idée de saleté associée à la couleur noire du chien, comme celle souvent évoquée par les mistis pour se référer aux paysans, jugés sans hygiène.

Contrairement au fonctionnement de la relation chien/homme, qui s'inverse après la mort, la relation entre mistis et paysans ne semble pas être altérée par l'identification des chiens psychopompes à ces deux catégories sociales et au rôle qui leur incombe. Comme si le renversement de l'ordre social était très improbable. Les rapports de force et de subordination se répètent jusque dans les visions de l'autre vie.

\section{Le séjour des morts dans la montagnelvolcan}

Après avoir traversé le fleuve, l'âme reprend sa route. Suivant les personnes interrogées, les lieux vers lesquels elle se dirige varient. Pourtant, tous ces espaces se ressemblent - ce sont les cols des plus hautes montagnes, les sommets enneigés... - et sont presque systématiquement associés à des volcans. Les toponymes mentionnés évoquent la géographie plus ou moins proche des locuteurs. Parmi les monts les plus fréquemment cités, on trouve le Coropuna (Qurpuna en quechua) - point le plus élevé du département d'Arequipa (6426 m) - ou encore l'Ausangate - montagne la plus haute du département de Cuzco $(6394 \mathrm{~m})$. Identifiables dans le paysage, ces lieux ne sont ni abstraits, ni totalement extérieurs au monde sensible et réel. Toutefois, ils se définissent par leur caractère liminal, car ils sont localisés à la périphérie des espaces domestiques. Sans pour autant représenter des endroits fictifs, ils sont inaccessibles aux hommes. En ce sens, ils appartiennent déjà à l'autre vie, raison pour laquelle il est précisé que seuls les morts peuvent les atteindre ${ }^{10}$. Le volcan apparaît comme un lieu de passage obligatoire des âmes, puisqu'il se trouve sur le chemin qui mène au pays des morts, ainsi que l'indique Pablo Alvis lorsqu'il commente la spécificité de la montagne Coropuna : «[Le Qurpuna] est un volcan. Mais je n'y suis pas allé, là-bas. Qu'est-ce qu'il peut bien y avoir? On raconte qu'une fois morts, nous irons sans aucun doute sur ce volcan. Il est certain que nous arriverons au volcan, au volcan du Qurpuna » ${ }^{11}$. La localisation du volcan vers lequel se dirigent les morts reste malgré tout un peu approximative et floue, tout comme la cartographie de l'autre vie. Plusieurs témoignages recueillis, aussi bien dans la province de Calca que dans celle de Chumbivilcas, mentionnent un volcan situé du côté d'Arequipa, zone de la cordillère occidentale, et de grande intensité volcanique. À Chumbi- 
vilcas, celui du Qurpuna est le plus fréquemment cité. Mais ceux qui en font la description ne s'y sont pas (encore) rendus, comme me l'a judicieusement rappelé Pablo Molina, lors d'une rencontre où je lui avais demandé trop de détails sur ces lieux menant à l'autre vie ! Ainsi, si les descriptions de cette géographie du monde des morts sont riches d'enseignements pour comprendre les projections des hommes sur leur devenir post-mortem, ne cherchons pas à déterminer des contours trop précis et irrévocables, ni à trouver des lieux immuables. Un même narrateur peut parfois évoquer un lieu, ensuite se corriger et parler d'un autre dont il se souvient, qui peut être situé à l'opposé, mais qui réunit les conditions qui lui semblent importantes pour le passage de l'âme. C'est ce qui ressort de l'explication d'Apolinaria Molina qui, dans un premier temps, situe le volcan à Arequipa, puis hésite à le placer sur le Salcantay - montagne $(6271 \mathrm{~m})$ du département de Cuzco, dans la cordillère orientale-, voire sur le Pichupichu - montagne $(5540 \mathrm{~m})$ au sud-est de la ville d'Arequipa : « Dans l'autre vie, comment ça peut bien être dans l'autre vie. [L'âme] doit d'abord passer par un volcan. C'est sûrement le volcan d'Arequipa. Ou est-ce que ça pourrait être le Salcantay ou Pichupichu? ${ }^{12}$. Ce volcan est souvent dépeint fumant, comme sur le point d'entrer en éruption. La fumée est un élément important, car elle est couramment utilisée comme un véhicule permettant d'établir la communication avec les êtres surnaturels. Son rôle dans la crémation des offrandes est crucial. Elle est en effet un support de contact et le moyen effectif d'envoyer la substance (animu) des offrandes aux entités concernées. On peut citer l'exemple de celles qui sont brûlées à l'intention des esprits tutélaires des montagnes durant les rites de fertilité des animaux, voire lors du rituel d'expulsion de l'âme qui conclut les funérailles. Les offrandes sont brûlées pour que leur essence immatérielle soit envoyée dans l'autre vie. Par ailleurs, le feu en puissance contenu dans les volcans est un autre aspect important de ces emplacements, car les morts qui s'y rendent sont calcinés ${ }^{13}$. Afin d'analyser la place de ce lieu dans le destin des morts, examinons les narrations sur le séjour de l'âme dans le volcan. Nous prendrons deux versions comparées : la première (V1) racontée par Pablo Molina (communauté de Lutto, Chumbivilcas) et la seconde (V2) racontée par Julián Poccohuanca (communauté de Pampallacta, Calca).

(V1) [Le mort] s'échappe vers le volcan. Et il s'assoit comme ça. Sur les bords de ce volcan, il y a des morceaux de fer qui brûlent. À cet endroit se trouvent les petits anges qui balaient. Ils balaient les cendres et les réunissent comme qui dirait là-bas. Et c'est comme ça qu'ils nous brûlent d'abord cette partie [Pablo désigne sa jambe gauche], puis ils nous brûlent celle-ci [Pablo désigne sa jambe droite] et finalement ils nous brûlent totalement. Ces petits anges jettent des rubans de chapeaux depuis le paradis. [[En disant " que [tel mort] ne brûle pas » ils suspendent [des rubans de chapeaux] à plusieurs endroits. Mais cette âme ne peut les atteindre, elle n'y arrive pas. Après plusieurs tentatives elle les attrape] ${ }^{14}$. Le mort s'accroche à eux, puis il les attrape. Il y a ce grand ruban. Alors [les petits anges] font s'échapper ceux qui se sont accrochés [au 
ruban]. [...] C'est comme ça que les gens disent : « tu vas d'abord brûler dans la maison du diable ». Et une fois que [le mort] a été libéré, je ne sais pas où il va.

(V1) Volcanmanqa escapasqa. Hinaspa ankhaynata tiyayusqa. Hinaspas tiyayuqtin cantonmanta chay volcanqa fierrokumas rawrayushan. Hinaspa chaypi angelitokuna pichashan. Hinaspa pichaspa usphallataña huñushan haqhaynaman. Hinaspas anchaymantas ankayllataraq ruphayuwanchis ankayllataraq ruphayuwanchis q'alata ruphayuwanchis. Chay angelitos hanaqpachamanta cintilluta wikch'uyamun. [[Hinaspas aqnata 'ama ruphachunchu' nispa aqnaman aqnaman aqnaman aqnaman warkuyamun. Hinaspa kay alma riki mana aypamuyta atinchu mana atinchu. Kaq aqnata aqnata chayqa hap'irum. ]] Hinaspas chayman hap'ipakun, aypapakun chay almaqa. Hinaspas chay hatun cintillu kan. Chaytaqa hap'iparukuspas chaymanta chay hap'ipakuqtaqa escapachin. [...] Anchaypis nisqa "supaywasipiraq rawranki" nispa rimasqaku runakuna. As anchaymantaña librakuspa riki maytas haykunpas riki? (Pablo Molina, annexe de Ch'alla Ch'alla, communauté de Lutto, Chumbivilcas, 17/06/1999)

(V2) Avec leur fourche, des diables poussent dans le feu les pécheurs, les morts qui ont des péchés. C'est là-bas que les morts brûlent. Ils y jettent les âmes pécheresses, les pécheurs, en les poussant. [Le mort] est malade en enfer, c'est comme les malades qui sont à l'hôpital et ne guérissent pas pendant plusieurs années, c'est pareil, c'est comme ça. Leur corps ne [...]. Dans cet enfer ils ne peuvent pas être en bonne santé pour la plupart. [L'âme] est comme brûlée par le feu, le feu à l'intérieur du four, le feu brûle mais il ne consume pas. [Plus loin dans l'enregistrement, Julián Poccohuanca revient sur cette histoire pour rajouter des éléments oubliés sur le sort des âmes dans le volcan]. Il faut élever de petits et de jolis chats. L'âme des chats rentre dans le volcan. [Là-bas] il y a les diables, propriétaires des fours, qui sont réunis et, dès qu'ils voient [les âmes], ils les brûlent. Alors le chat enfonce rapidement sa main à l'intérieur [du four]. Dès qu'il voit un homme, le chat enfonce sa main à l'intérieur [du four pour l'attraper] et le jette au loin d'un coup. Les diables ne s'en aperçoivent pas. Après l'avoir fait sortir du four, [le chat] l'envoie au loin. Il arrive et tout à coup jette [l'âme] au loin. C'est comme ça qu'il en sauve. Ce sont les pécheurs qui sont là, dans le volcan.

(V2) Chaysi Satanaskunas huchayuq huchayuq runata wist'ashan tankawankama chay yawrasqapipas. Chaypis alma t'iktiyushan. Tanqa tanqawansi chanqayun chay huchayuq almata huchayuqta. [...] Unqushan infierno infiernopi, hospitalpi unqushan mana watan watan mana qhaliyaq kaqlla, hinalla. Yasta manaña kurpun... manas chay infiernopi kaq allinraqchu aswan riki. Chay nina ruphasqan kaqllayá, nina horno sunqupi nina ruphashanchá mana mana yawranchu. [...] Chaysi michichatapas uywakuna, munasqachata. Michiq riki alman volcanman haykun riki. Rikurquspa huñuyusqa chay satanaskuma kanashan anchayna hornoyuqkuma kashan. Chaysi michiqa hukta haywaykusqa. Chay runata rikurqun chaypachaqa haywaykuspas huk makinwan " t'aq " chanqarqun karuman. Chaysi Satanaskuna mana rikunchu. Karumansi chanqarqun hurquruspa hornomanta. Qunqaylla hamuruspa chanqaramun. Chayqa liwransiyá chayta. Hina chayqa chay volcanpi chay huchayuqkunas chaypi kashan. (Julián Poccohuanca, communauté de Pampallacta, Calca, 18/09/1998) 
On remarque que les noms associés à ces volcans relèvent du vocabulaire infernal et du domaine du diable. On parle d'un " enfer » (infierno) peuplé de " diables ", appelés satanas, dans V2 et de la maison du diable (supaywasi) dans V1. Dans la communauté de Khuyo Grande (province de Calca, département de Cuzco), les cratères des volcans sont également désignés comme des voies d'accès à l'enfer (Casaverde 1970, p. 207). Pourtant, notre volcan apparaît bien comme un lieu intermédiaire, entre le monde des vivants et celui des morts, que l'âme n'a pas encore atteint. Ce séjour est une étape transitoire pour l'âme. Dans V1, la particule de l'inaccompli -raq (dans la phrase " supaywasipiraq rawranki », " tu brûleras d'abord dans la maison du diable ») indique sans aucun doute possible que le séjour dans la maison du diable précède le départ vers un autre lieu où l'âme se rendra ultérieurement. Il est bien précisé qu'elle y brûle temporairement. Avant de sortir du volcan, elle doit d'abord y brûler. D'ailleurs, "l'enfer », comme lieu de séjour temporaire des âmes dans leur voyage vers l'autre vie, se retrouve dans la communauté de Cotabamba (province de Calca, Cuzco). Les âmes envoyées au supaywasi par des démons (demonios) n'y restent qu'un temps avant d'en sortir (Nuñez del Prado 1970, p. 111). Au terme de ce laps de temps, l'âme peut enfin s'échapper. Elle est alors « libérée " (librakusqa), dans V1, par les anges et, dans V2, par les chats. La difficulté des âmes à atteindre les rubans suspendus, dans V1, montre qu'elles ne sont pas encore prêtes à sortir du volcan. Comment comprendre cet échec préliminaire ? Cet épisode évoque-t-il l'attente nécessaire à la destruction des péchés que contiendrait l'âme ? Très certainement puisque cette idée de péché est explicitement formulée dans V2. Les âmes qui arrivent sur le volcan sont toutes pécheresses (huchayuq) ${ }^{15}$. Ensuite seulement, elles sont sauvées par les chats qui les extraient du feu, à l'instar des chiens décrits plus haut. La durée du séjour dans le volcan est proportionnelle à la quantité des péchés de l'âme. Sans leur disparition préalable, la poursuite de son trajet n'est pas possible. C'est ce que montre cet autre commentaire de Julián Poccohuanca, postérieur à son récit V1, et qui est encore plus précis sur ce point : « Lorsque nos péchés sont consumés par la cuisson [...]. Il paraît [que dans le volcan, le mort] fume pendant des années. Il fume à cause de ses péchés [...]. Il se consume par cuisson, puis s'envole par la porte du four sous la forme d'une colombe blanche ${ }^{16}$. Les brûlures cessent après que les péchés ont été « cuits » dans le volcan, leur élimination progressive se matérialisant par la fumée qui se dégage du mort. Le feu contenu dans les volcans est aussi expiatoire, puisqu'il permet la libération finale de l'âme. Il semble nettoyer l'âme, il la purifie. D'ailleurs, l'allégorie de la colombe, courante dans le christianisme pour représenter les « âmes des justes » (Amat cité par Carozzi 1994, p. 209), intervient ici comme la manifestation de la rémission des péchés et du salut de l'âme. Bien qu'elle soit brûlée, l'âme continue à vivre car ce n'est pas elle qui est détruite par le feu, mais ses péchés : «C'est par la volonté de Dieu que le mort vit, même brûlé. Ensuite, il part vers le pays des morts " ${ }^{17}$. La durée de combustion de l'âme dépend de la décision divine. C'est Dieu qui l'autorise à 
gagner le pays des morts, une fois sa purgation achevée. L'emplacement de ce lieu, où vont finalement tous les morts, est explicitement situé dans le hanaqpacha (terme quechua pour désigner le paradis) : « Je ne sais sous quelle forme, après s'être libérée, l'âme grimpe au paradis vers Dieu. Quand elle a fini d'y brûler [...], c'est à cause de ses péchés qu'elle brûle là-bas [dans le volcan]. Ensuite elle est probablement absoute, je ne sais pas. Après seulement, elle est libérée ${ }^{18}$.

Si le feu du volcan constitue le moyen le plus usuel de délivrance des morts, certaines versions associent la libération de l'âme à une croix située sur une montagne qu'elle doit atteindre. Néanmoins, l'élément primordial attaché à ce lieu consiste toujours en l'élimination des péchés du mort. Généralement, ces récits opèrent une analogie explicite entre le sort de l'âme et celui du Christ, thème présent dans certaines prières de neuvaine qui établissent une relation directe entre le sort posthume de l'âme et les étapes successives du martyre du Christ (voir par exemple la Novena muy devota... de Galindo 1855). Le salut de l'âme s'obtient donc après une véritable via crucis :

[L'âme] va sur l'Ausangate. Les anciens racontaient qu'« il y a une croix de fer et qu'à cet endroit nos péchés doivent d'abord être absous ; c'est là que nous sommes crucifiés ; ensuite nous pleurons énormément » disaient-ils. Comment Dieu nous juge-t-il ? Comment ? [...] Combien ? ${ }^{19}[\ldots .$.$] Les morts s'en vont vers le volcan. C'est là qu'ils sont$ d'abord, sur le volcan. Ensuite, ils se transforment en colombe, pour pouvoir s'envoler, juste à la sortie [du volcan] à l'endroit où se trouve la sainte croix, pas à l'intérieur du volcan.

Awsangateta rin riki. Nispan niqkuñawpakunaqa " fierro kurusmi kashan ; chaypiraqmi imapas huchanchisqa pampachakun ; chaypi chakatakunchi s; hinaspas chakatayuspas waqayusunman " ninkun riki. Chayqa imayna Dios sentenciawasunman riki, imaynan hayk'an [...] Volcanman almakuna rishanku. Chaymanraq chayanku volcanman. Chaymantañataq palomaman wakipunku phawarikapunanpaq hawallapi chay santakuruspa kasqanmantas riki manan volcan ukhupichu. (Gabriela Vera, annexe de Ch'alla Ch'alla, communauté de Lutto, Chumbivilcas, 20/03/1999)

Ainsi, il apparaît que le voyage de l'âme vers l'au-delà se justifie par les péchés que porte tout mort, comme l'attestent aussi certaines narrations sur le Jugement divin après le décès (Robin 2002, pp. 109-113). C'est du reste la raison pour laquelle l'enfant baptisé, mort en bas âge et considéré sans péché (angelito), n'est, quant à lui, pas censé effectuer ce périple post-mortem. Il se rend directement au hanaqpacha, au paradis. Les funérailles de ces enfants, souvent peu élaborées, se déroulent sur un laps de temps plus réduit que pour les adultes. En effet, il n'y a pas à réaliser de préparation rituelle au voyage vers l'au-delà. C'est pourquoi, à Lutto comme à Pampallacta, après l'enterrement de jeunes enfants aucun rite d'expulsion de leur âme n'est effectué.

Une fois entré au paradis, le transit du mort est révolu. Cette incorporation de l'âme dans le village des morts clôt le processus de fabrication du mort. Si le séjour 
dans le volcan évoque un "enfer» - ce que l'on retrouve dans les différents termes utilisés pour le désigner -, nous avons également vu qu'il ne s'agit que d'un lieu de passage temporaire, purgatoire. Examinons maintenant plus précisément la symbolique de la montagne/volcan pour comprendre sa signification au sein du voyage du mort dans l'autre vie. Intéressons-nous en premier lieu aux interprétations qui ont couramment été données à ce sujet.

\section{RÉFLEXIONS SUR LA GÉOGRAPHIE DE L'AU-DELÀ}

\section{Survivance préhispanique et influence indigéniste}

Certains anthropologues, travaillant dans le Sud du Pérou, ont analysé les conceptions actuelles de l'au-delà et du voyage des morts comme des survivances de représentations préhispaniques. Ils souhaitaient démontrer que les rites funéraires auxquels ils avaient assisté et les récits sur le voyage du mort qu'ils avaient recueillis étaient le reflet de conceptions antérieures à l'évangélisation coloniale. Les cérémonies issues des temps préhispaniques, à quelques exceptions syncrétiques près, continueraient à se pratiquer de nos jours et prouveraient « une fois de plus la résistance et la capacité de lutte de la culture et du peuple andins ${ }^{20}$. D'autres auteurs estiment que les membres des communautés andines continuent à célébrer leurs défunts sans grand changement par rapport à l'époque inca, même s'ils notent quelques modifications dues à l'implantation du catholicisme (Rosell 1976). Cette thèse, parce qu'elle est courante, mérite qu'on s'y attarde un instant car ses assertions posent un problème épistémologique et les observations que j'ai faites sur le terrain semblent la contredire radicalement.

Pour nombre d'auteurs, la présence même du volcan Qurpuna dans les représentations actuelles de l'au-delà constituerait la preuve irréfutable de réminiscences préhispaniques, puisque les chroniques coloniales désignent les volcans comme lieux de séjour des morts. On peut citer le chroniqueur Guaman Poma de Ayala (1987, p. 290). En 1613, il écrit que, pour les populations précolombiennes du Collasuyu et de Condesuyo ${ }^{21}$, " les défunts allaient directement à Puquinapampa et à Coropuna ». Les chroniques mentionnent également des données intéressantes sur les croyances païennes héritées des temps préhispaniques, notamment en ce qui concerne le destin posthume des défunts. On peut signaler le texte du jésuite Arriaga, La extirpación de la idolatria en el Pirú. Cette extraordinaire « ethnographie » de 1621 constitue en fait un manuel visant à enseigner aux futurs curés les « idolâtries » des Indiens pour mieux les combattre et, par la suite, imposer la foi chrétienne. Arriaga (1999, p. 76) - dont l'ouvrage résume les rapports des visites d'idolâtrie menées entre 1619 et 1621 , auxquelles il a activement participé - indique qu'après le décès, l'âme du mort doit traverser un fleuve grâce à des chiens noirs, ce qui explique les mises à mort sacrificielles de ces 
animaux au moment des funérailles. Certes, ces informations ne concernent pas l'époque préhispanique et les « idolâtries » des Indiens au XVII ${ }^{\mathrm{e}}$ siècle et la réalisation de ces rites n'avaient peut-être plus tout à fait la même signification qu'au siècle précédent. Il est aussi vrai qu'un sacrifice de chien avait bien lieu dans le contexte funéraire préhispanique. Sa dépouille était ensevelie avec le cadavre, comme l'atteste la fameuse tombe du Seigneur de Sipán (Lambayeque), laissant entrevoir ainsi son rôle de psychopompe. De même, l'iconographie des poteries mochica atteste la présence du lama dans les pratiques mortuaires (Bourget 1996, pp. 44-45). Cet animal était également immolé et enseveli avec le défunt. On en trouve notamment dans les tombes des dignitaires du royaume chimú de Chan Chan, ainsi que dans celles de Sipán, sur la côte nord-péruvienne, et jusque dans la puna de Catamarca en Argentine (Pablo Cruz com. pers.).

Mais une question reste en suspens. Si certains éléments de l'au-delà actuel ont très certainement été puisés dans une tradition antérieure à la colonisation espagnole, la quête de substrats préhispaniques n'a-t-elle pas eu pour effet d'occulter la réélaboration chrétienne qu'ils ont pu et dû subir durant l'évangélisation ? Les détails jugés trop chrétiens ne sont-ils pas laissés de côté par ceux qui les considèrent superficiels? Tenues pour quantité négligeable, les composantes dites chrétiennes sont alors détachées d'un ensemble perçu comme véritablement autochtone - c'est-à-dire préhispanique ${ }^{22}$. L'objectif de plusieurs auteurs semblent être en effet de démontrer que des traditions précolombiennes survivent dans des domaines essentiels de la culture paysanne contemporaine et de minimiser, voire de nier, l'impact de la christianisation qui n'aurait jamais été véritablement acceptée. Les interprétations qu'ils proposent demeurent cependant problématiques, puisque l'allusion au christianisme est présente malgré tout.

Il n'est peut-être pas inutile de rappeler ici que nombre d'anthropologues de Cuzco participent activement au processus d'ethnogenèse « néo-inca » ou fondée sur l'" andinité » (lo andino) qui s'élabore, depuis plusieurs années, dans cette ville des Andes du Pérou (Antoinette Molinié com. pers.) et prend explicitement ses racines dans l'indigénisme ${ }^{23}$. Pour justifier leur position, les anthropologues cuzquéniens ont besoin de montrer scientifiquement que les paysans andins ont résisté culturellement. Sans cela, ils ne pourraient rechercher en eux l'« andinité authentique » dont l'entreprise indigéniste a besoin pour construire une culture et une identité régionales ou nationales fondées sur l'autochtonie, en rupture avec les apports occidentaux. Or le culte des morts ou des ancêtres est aujourd'hui l'une des caractéristiques les plus marquantes de la société préhispanique, telle que l'archéologie et l'histoire l'ont reconstruite. Retrouver dans le rapport aux morts l'héritage presque intact du passé précolombien s'avère particulièrement important pour l'affirmation d'une continuité culturelle ${ }^{24}$. Par ailleurs, ces interprétations soulèvent un autre problème. Sous couvert d'un discours apologétique, l'image des paysans quechuaphones, qui ressort de ces études, est celle de personnes ayant une identité qui serait restée figée dans un passé préhispanique. Cette 
tradition - dont seuls les paysans les plus éloignés des centres urbains seraient les dépositaires -, tout en servant de modèle à un projet de société future fondé sur $l o$ andino, contribue concrètement à accentuer l'idée d'altérité attachée aux paysans andins. Les membres des communautés semblent réifiés dans une réalité qui " paraît arrêtée dans le temps » ${ }^{25}$, comme s'ils s'étaient maintenus à l'écart de la société nationale, insensibles aux changements de l'Histoire depuis la Conquête !

Cependant, suffitit-il de déceler des éléments de la vision de l'au-delà préhispanique, mentionnés dans des textes coloniaux, pour affirmer qu'il y a bien maintien des catégories mentales constitutives de la pensée précolombienne ${ }^{26}$ ? Sans nier le poids des héritages, il ne faut pas réduire les faits culturels à leurs origines supposées, mais montrer comment ils ont été assumés par les sociétés à chaque moment de leur histoire, pour aller dans le même sens que l'historien Jean-Claude Schmitt (1993, p. 253). Il est donc nécessaire de ne pas minimiser, voire de ne pas oblitérer, le processus historique depuis la période coloniale et de prendre plus sérieusement en considération la christianisation qui représente, dans les Andes, une étape essentielle pour comprendre les représentations actuelles de l'au-delà. C'est pourquoi il est indispensable de s'intéresser à la mise en place des politiques évangélisatrices et de s'interroger sur le rôle joué par l'Église dans l'élaboration des récits eschatologiques à travers l'investissement de l'imaginaire de l'au-delà des Indiens à l'époque coloniale.

Ainsi, je voudrais montrer que les représentations actuelles du périple posthume de l'âme sont imprégnées non pas de concepts préhispaniques, mais, bien au contraire, de l'idée du purgatoire catholique. Son influence dans la formation de la géographie actuelle de l'autre vie est centrale et, tout particulièrement, explicite dans le cas du séjour dans la montagne/volcan.

\section{Le volcan comme purgatoire infernalisé andin}

\section{Retour sur le purgatoire en Europe}

La « naissance du purgatoire », pour reprendre le titre de l'ouvrage de Jacques Le Goff (1981), est en partie liée à l'intérêt croissant pour la situation des âmes entre la mort individuelle et le Jugement dernier ${ }^{27}$. La croyance en un double jugement - le premier au moment de la mort, le second à la fin des temps - a contribué à la genèse de ce lieu dans la cartographie de l'au-delà chrétien. L'instauration du purgatoire comme lieu établi date approximativement du début du XIII ${ }^{\mathrm{e}}$ siècle. Son existence est assumée comme dogme à partir du Concile de Lyon de 1274. Jusqu'à cette époque, le pouvoir judiciaire spirituel, soit le tribunal de l'âme, était nettement séparé par la frontière de la mort. Postérieurement, l'Église affirme son droit sur les âmes du purgatoire, poussant en avant le for ecclésiastique au détriment du for de Dieu, pourtant détenteur de la justice dans l'au-delà. L'apprivoisement de l'au-delà, que permet le purgatoire, ajoute les 
morts à l'encadrement général de la société. Dans sa situation d'intermédiaire entre le paradis et l'enfer, il permet un accroissement de l'emprise de l'Église sur les fidèles en prolongeant son pouvoir par-delà la mort, car c'est elle qui administre ou contrôle les offrandes de toutes sortes (prières, messes, aumônes), accomplies par les vivants en faveur de leurs morts, et elle en tire bénéfice. L'image du purgatoire s'est progressivement détachée de l'enfer par son aspect passager pour se rapprocher du paradis qu'atteindraient les âmes, une fois leurs peines expiées. Elle a toutefois reproduit partiellement l'imagerie infernale et l'épreuve par le feu a joué un rôle de premier plan : l'histoire du purgatoire montre que ce lieu s'est développé comme un " modèle judiciaire ». Ainsi, le feu du purgatoire est devenu un enfer temporaire destiné à la purgation des péchés (Carozzi 1994, p. 265). Pour Saint Thomas d'Aquin, c'est d'ailleurs un même feu qui brûle en enfer et au purgatoire (Fournié 1997, p. 488).

\section{L’implantation du purgatoire au Pérou}

Durant les années suivant la conquête du Pérou, l'absence du purgatoire vaut pour tous les textes de catéchèse antérieurs à 1583. Il s'agissait probablement, comme en fait l'hypothèse Juan Carlos Estenssoro (2003, pp. 251-252, 409), de contribuer à éliminer les anciens cultes des morts. Avec l'enfer et le paradis, il n'y avait en effet pas de place possible pour l'action des vivants sur leurs morts. Le purgatoire fit son apparition oflicielle au Pérou à partir du Troisième Concile de Lima (1582-1583) qui élabora des textes de catéchèse en quechua et en aymara dans lesquels cet endroit était désigné par le mot castillan purgatorio. La dévotion concernant les âmes du purgatoire fut particulièrement encouragée par l'Église coloniale. Comme dans le reste du monde catholique après le Concile de Trente (1562), la diffusion de pratiques cultuelles pour les âmes du purgatoire acquit, au Pérou, une importance qui se manifeste dans la création de plusieurs confréries. Le purgatoire permettait aux hommes d'agir sur leurs morts et, réciproquement, ces derniers pouvaient intervenir et modifier le sort des vivants. Ce double jeu, légalisé par le dogme, était connu de tous.

Dans son manuel d'évangélisation, Symbolo católico indiano (1598), le franciscain Luis Jerónimo de Oré insiste sur la nécessité de développer le culte des âmes du purgatoire, tout en dénonçant avec virulence les prêtres qui ne faisaient pas respecter la célébration des oraisons et des messes à l'intention des trépassés :

[Les pères doctrinaires] verront par expérience la nécessité de ce secours car les Animas n'en ont pas d'autre, la rosée des prières de l'Église et du peuple chrétien, avec laquelle sont soulagés et rafraîchis dans les flammes de leur tourment, ceux qui n'ont pas été complètement satisfaisants par les fautes [commises] dans cette vie, jusqu'à ce que Dieu les sorte de leur peine et les emmène dans sa Sainte Gloire. (Oré 1982, chap. XV, « De la devoción de las Animas de purgatorio », pp. 192-193) 
On retrouve ici le feu propre au purgatoire infernalisé. Grâce aux suffrages de sa famille, l'âme obtient la possibilité de se sauver et voit la durée de ses souffrances dans le purgatoire se raccourcir pour accéder enfin au paradis où Dieu l'acceptera. Cette attention aux âmes du purgatoire semblait d'ailleurs si importante à Luis Jerónimo de Oré qu'elle sera à nouveau mentionnée dans son testament (Pello 2000).

En Europe, les exempla ont servi de moyen de diffusion de masse pour le développement du purgatoire (Le Goff 1981). Ces récits édifiants ont également été répandus dans le Nouveau Monde, à des fins de prédication durant la période coloniale $^{28}$. Il serait intéressant de savoir si ce type de récits a servi au Pérou à propager le thème du purgatoire parmi les nouveaux convertis, comme ce fut le cas pour le Mexique (Dehouve 2004). Les prédicateurs catholiques n'auraient-ils pas pu reprendre et réutiliser l'image préhispanique du volcan en la faisant coïncider avec le purgatoire ? On sait que cette politique de substitution était courante durant la première évangélisation, caractérisée par la recherche de continuité entre les rituels indigènes et catholiques (Estenssoro 2003, Partie I). Rappelons aussi que, dans les tentatives de localisation terrestre du purgatoire, le volcan, comme élément géographique naturel, avait particulièrement retenu l'attention de l'Église médiévale. Il avait l'avantage de rassembler trois des éléments essentiels de la structure physique et symbolique du purgatoire : la montagne, le cratère, le feu (Le Goff 1981, p. 20). De plus, l'Église évangélisatrice, si préoccupée par l'enracinement du culte des ancêtres dans les Andes, n'intervenait-elle pas concrètement sur ses fidèles en investissant cet imaginaire du destin des morts vers l'au-delà ? Cela ne lui permettait-il pas d'augmenter son contrôle sur ses ouailles, comme ce fut le cas de la politique qu'elle mit en place en Europe?

\section{Commentaires actuels sur le purgatoire}

Aujourd'hui, sans l'associer directement au volcan, plusieurs personnes se réfèrent au purgatorio comme un lieu de feu ${ }^{29}$ où séjournent les morts pécheurs. Si ce terme n'est pas aussi couramment évoqué dans le discours sur l'au-delà que peuvent l'être ceux qui qualifient, depuis le $x v I^{\mathrm{e}}$ siècle, l'enfer ou le paradis, le purgatorio est malgré tout connu dans la région de Cuzco ${ }^{30}$. Les doutes sur sa localisation, voire parfois l'oubli de son existence, sont certainement dus à son absence des catéchismes depuis, au moins, la seconde moitié du $\mathrm{xx}^{\mathrm{e}}$ siècle (Xavier Pello com. pers.). Mais, le souvenir d'un lieu appelé purgatoire reste plus ou moins présent dans les esprits. À l'occasion de la Toussaint, certaines des oraisons récitées par les diseurs de prières dans le cimetière comportent toujours la mention du salut des âmes du purgatoire.

Les similitudes entre le volcan où se rendent les âmes et le purgatoire comme lieu de rédemption et de rémission des pêchés sont frappantes. À tel point que le 
jour où, à Lutto, je discutais avec Pablo Molina du purgatoire, il se mit à réfléchir en doutant quelques instants. Puis, ne sachant plus réellement à quoi correspondait le purgatoire mais estimant que ce mot lui disait quelque chose, il me demanda, apparemment par association d'idées, s'il ne s'agissait pas du volcan. Il me relata à nouveau sa vision du parcours des âmes dans le volcan (avec quelques variations par rapport à V1), mettant cette fois ce dernier en parallèle avec le purgatoire. Je précise que ma question sur le purgatoire n'a pas fait suite à des commentaires préalables sur le volcan qui auraient pu évidemment influencer sa réponse :

V.R. : Comment est le purgatoire ? Les âmes vont-elles au purgatoire ?

- Au Purgatoire [Vergatorio (sic.)]. Qu'est-ce que le purgatoire? C'est le volcan ou qu'est-ce que c'est ?

V.R. : Est-ce que le volcan est le purgatoire?

- Oui l'âme s'en va vers le volcan oui. Sur le volcan, il y a une croix en fer. Alors [les âmes] se dirigent les unes derrière les autres vers le sommet. Lorsque le soleil décroît et commence à se cacher, la croix de fer se met à brûler et à faire de la fumée. C'est à cet endroit que les petits anges rassemblent les cendres des âmes. C'est comme ça que les âmes s'approchent du purgatoire. Alors [le feu] brûle d'abord un côté, ensuite un autre [de l'âme], puis entièrement. Finalement, les petits anges envoient des rubans [de chapeaux] vers les mains [des âmes] comme ça (Pablo mime les fils suspendus par les petits anges que les âmes doivent attraper). Ensuite [les anges] portent les âmes dans leurs bras et les sauvent. C'est peut-être ça que vous appelez le purgatoire ? C'est là que [les âmes] se sauvent. S'ils n'attrapent pas [les rubans] [le feu] les brûle entièrement.

V.R. : Imaynataq Purgatorio ? Almakuna purgatorioman rinkuchu?

- Vergatorioman [sic.]. Vergatorioqa imataq kanman? Volcan? Ima?

V.R.: Volcanchu purgatorio?

- Riki chay almaqa rin riki volcanman riki. Volcan chaypitaqsi fierro kurus kashan. Anchaymantaqsi siq'i rishan riki rishan pataman. Hinaspa inti ña bajayamuq chinkayapushaqtinña chaypitaqsi fierro kurus rawrashan q'usñishan. Hinaspa anchayman angelitochakuna wañun anchaytaqsi ushphallataña almataqa chay usphanta huñum. Hinaspas ankhaynata almakuna chay vergatoriomanqa achhuyunku. Hinaspas ankay cantollantaqa cantollantaqa ruphan ruphan ruphan q'alata. Chayqa chay angelito reatilluta churanku chay makichanman riki aqnata. Hinaspa aqna chay almata chay marq'asqanku aqnata qispiyushanku. Anchaytachus ninkichis vergatorio nispa. Chaypi librakun. Sichus mana chayqa hap'ipakun chaypachaqa q'ala q'alachatasyá ruphan. (Pablo Molina, annexe de Ch'alla Ch'alla, communauté de Lutto, Chumbivilcas, 27/07/2002)

Certaines données, évoquées plus haut dans la description de l'intérieur du volcan, montrent des similitudes avec les représentations picturales du purgatoire. C'est le cas de l'épisode des anges qui envoient des rubans pour sauver l'âme du volcan et qui fait penser au cordon de Saint François, accessoire efficace pour tirer les âmes du purgatoire, que l'on retrouve en Espagne et dans l'Europe méditerranéenne, à partir du XvII ${ }^{\mathrm{e}}$ siècle (Vovelle 1996, p. 158). Des fresques 


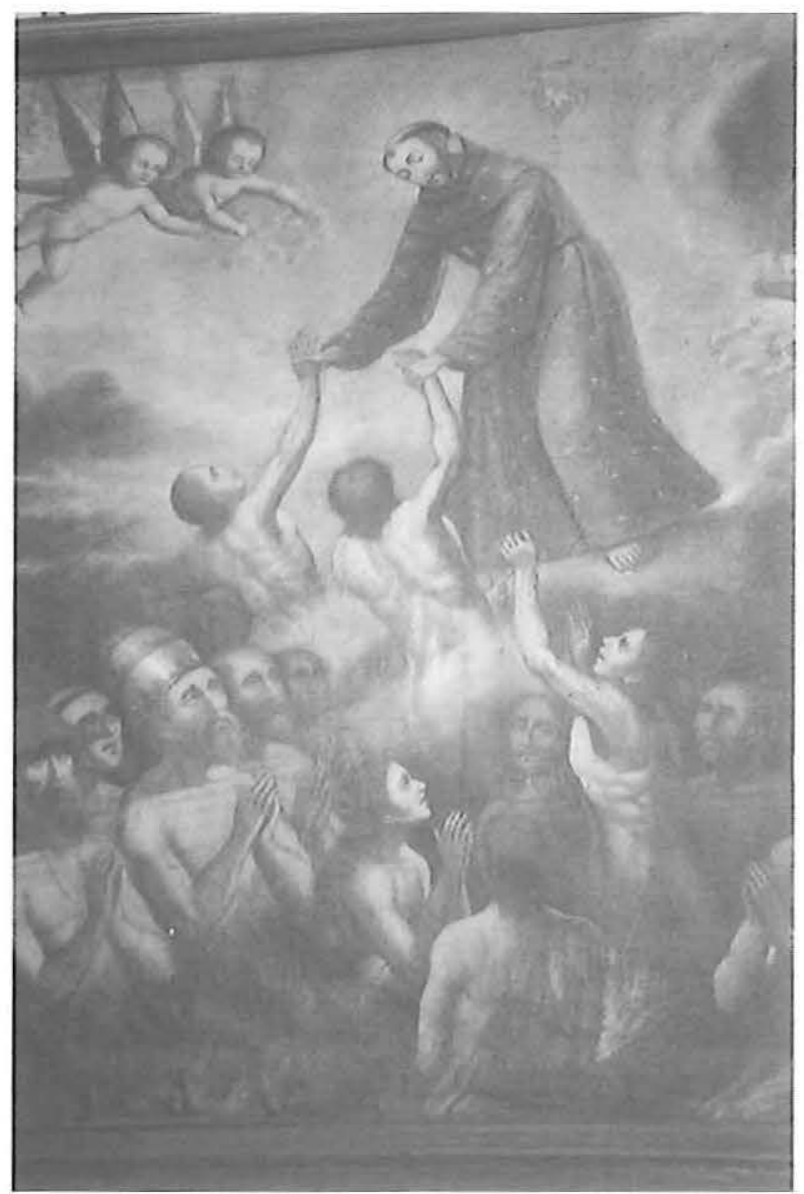

FIG. 2 - Âmes du purgatoire s'accrochant au cordon de Saint François pour obtenir leur salut. Couvent franciscain de Ocopa, département de Junin, Pérou (cliché Robin)

reproduisant cette scène ont aussi été diffusées au Pérou, puisqu'on en trouve la trace, par exemple, dans le couvent franciscain d'Ocopa (département de Junín) (Figure 2). De plus, les images d'anges extirpant les âmes du feu du purgatoire font partie de l'iconographie classique de ce thème. La peinture murale sur le Jugement dernier de l'église de Huaro (province de Quispicanchis, département de Cuzco) offre un des multiples exemples andins de la représentation du purgatoire avec des anges tirant les âmes du feu (Figure 3). Le séjour des âmes dans le four diabolique (mentionné plus haut dans V2) évoque les fresques de scènes infernales. Mais il renvoie aussi aux descriptions du purgatoire, comme celles que 


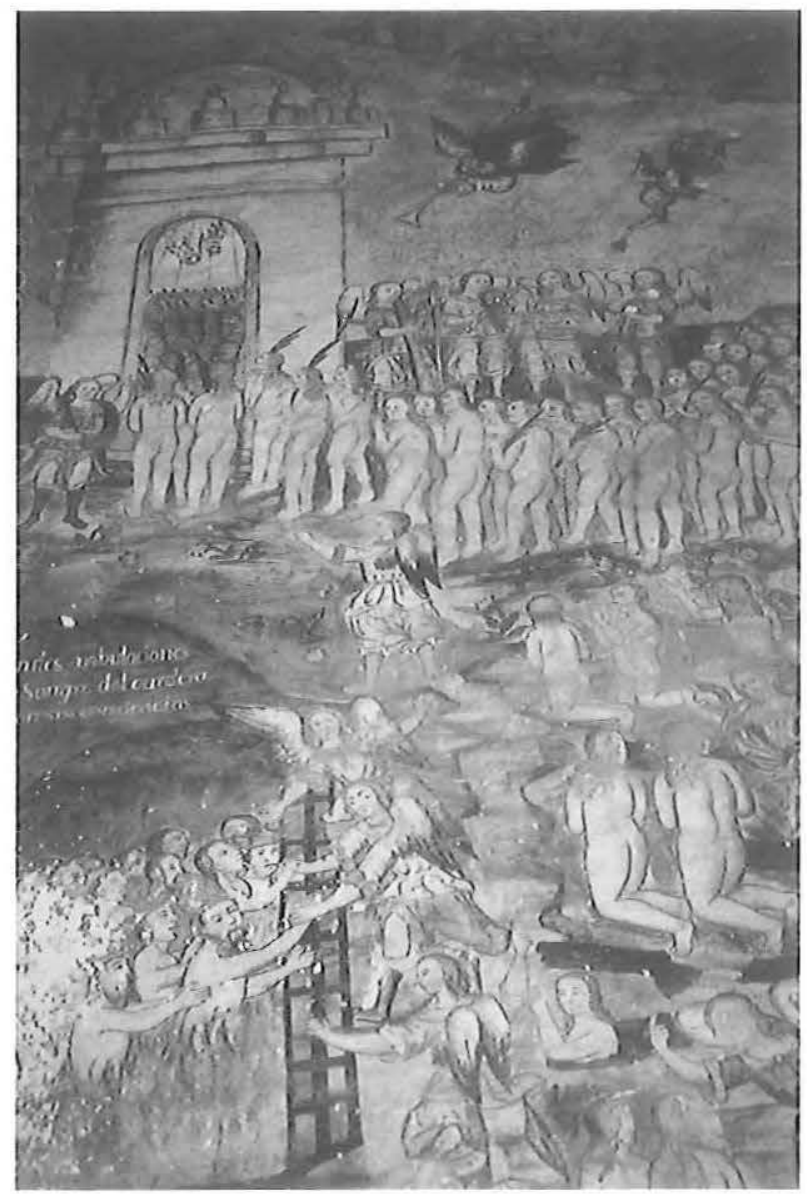

FIG. 3 - Anges sauvant les âmes du feu du purgatoire. Détail du Jugement dernier, peinture murale de l'église de Huaro, département de Cuzco, Pérou, par Tadeo Escalante, début du xix ${ }^{\mathrm{e}}$ siècle (cliché Robin)

l'on trouve, par exemple, dans le sermon XXX du Tercero Cathecismo de 1585 (Tercer Concilio Limense 1867, pp. 370-373) qui, par ailleurs, spécifie que le séjour en son sein concerne tous les morts, y compris les "bons chrétiens » : « Dieu fait entrer les bons chrétiens dans le four du purgatoire pour qu'ils soient purifiés de leurs péchés (Diosninchicpas alli Christianocunacta [...] huchanmanta llumpac cancanpac [...] Purgatoriopi [...] callanamanmi paycunacta cachay(uin) 》. 
Ainsi, aucune des données recueillies dans les récits eschatologiques individuels ne suggère que la conception actuelle du volcan reflète ou perpétue celle qui était en vigueur à l'époque préhispanique. En revanche, les explications fournies sur cette montagne, comme séjour de pénitence temporaire de l'âme, permettent d'avancer l'hypothèse que le volcan actuel où se rendent les morts correspond à une version " andinisée » du purgatoire infernal, tel qu’il a été apporté par les évangélisateurs. Hypothèse qu'il conviendrait de corroborer par une étude historique approfondie sur le prêche du purgatoire au sein de la population andine à l'époque coloniale.

\section{La victoire du purgatoire sur l'enfer : échec ou réussite missionnaire ?}

Revenons sur les termes employés pour désigner le volcan. Nous avons vu qu'il s'agissait d'un «enfer». En fait, dans le parcours vers l'autre vie, les différents termes pour "enfer» (ukhupacha, supay wasi, infierno) évoquent exclusivement un séjour transitoire des âmes. Ainsi, la localisation de cet " enfer », qui fait référence à des lieux situés à la périphérie des espaces socialisés (volcans ou sommets de montagnes), est terrestre et ne renvoie pas à un monde invisible, ni forcément souterrain. De façon surprenante, et alors même que de terrifiantes représentations picturales de l'enfer ont largement été diffusées dans les Andes (Figure 4), ce lieu a fini par être assimilé à un lieu de passage temporaire des morts. Il est vrai que, dans la géographie coloniale de l'au-delà, le purgatoire est une partie de l'enfer (Estenssoro 2003, p. 68) et le Cathecismo mayor signale que les tourments qui y sont endurés sont une réplique de ceux de l'enfer (Bouysse-Cassagne 1999). Dans son catéchisme de 1649, Jurado Palomino décrit le purgatoire comme un des quatre enfers, avec les limbes, l'enfer des patriarches et celui des damnés. Quant au sermon XXX du Tercero Cathecismo (Tercer Concilio Limense 1867, pp. 370-377), dans lequel sont exposées les souffrances endurées par l'âme, d'abord au purgatoire puis en enfer; on observe que la description faite de ces deux lieux est quasiment identique, avec une place prépondérante accordée au purgatoire. Il n'est donc pas très étonnant de penser que l'enfer et le purgatoire ont été confondus, en conservant l'idée de purgation qui caractérise le « $3^{\mathrm{e}}$ lieu ». Même si le nom d'enfer est mentionné dans les récits eschatologiques recueillis, ce lieu reste aujourd'hui très associé à la notion de purgatoire infernalisé, puisque tous les morts peuvent s'en délivrer finalement, même les morts qualifiés de « damnés ».

Les âmes en peine se caractérisent précisément par les lourds péchés accumulés durant leur existence (inceste, avarice ou encore actes de violence à l'encontre de parents). Les damnés ne peuvent accomplir leur trajet vers l'autre vie, comme les morts ordinaires. Ce sont des revenants au statut liminal qui errent anormalement dans le monde des vivants. De nombreux récits indiquent que ces morts peinent sur les cimes des montagnes ou dans la puna. Ces lieux, souvent dénom- 


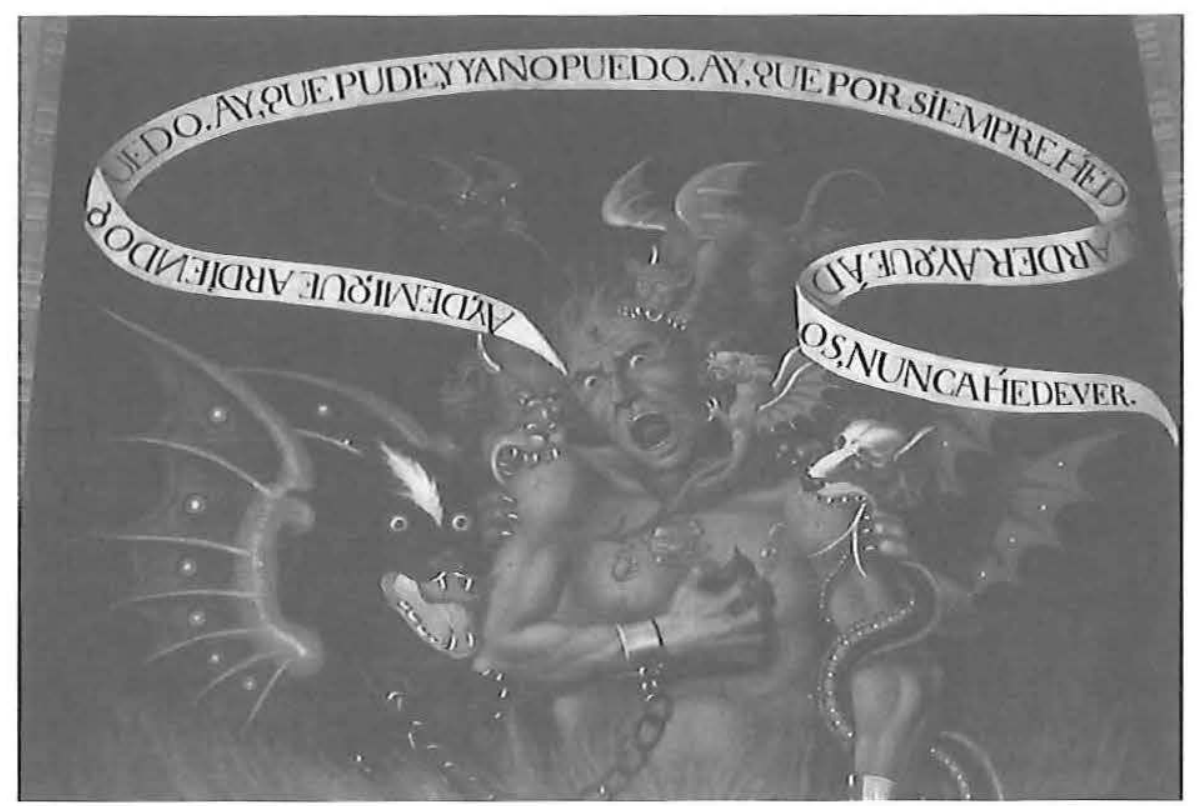

FIG. 4 - Âme damnée en enfer, Couvent mercédaire, Cuzco, Pérou, XvII siècle (cliché Robin)

més ukhupacha, sont comparés à une prison où les damnés sont en détention, à priori à perpétuité. Par ailleurs, ils sont contraints de revenir sur les lieux où ils ont commis leurs fautes afin de les expier, sorte de purgation terrestre. À l'instar des exempla médiévaux sur les revenants européens, qui cherchaient à susciter la peur (Schmitt 1993, p. 149), les témoignages sur le dérèglement engendré par les damnés constituent un discours indubitablement moralisateur sur les comportements répréhensibles. C'est ce qui ressort clairement des propos d'un damné qui apparaît aux hommes : « Ne soyez pas comme moi. Je frappais ma mère, je lui arrachais les cheveux. Ne soyez pas comme moi autrefois. C'est pour ça que Dieu m'a rejeté [dit le damné] ${ }^{31}$. Ce retour auprès des vivants est éminemment prédateur. La rencontre avec les damnés est toujours synonyme de malheur (maladie ou calamités météorologiques). La « spécialité » du damné est de dévorer les hommes trouvés sur son chemin et les descriptions de scènes d'anthropophagie sont légion. D'aucuns diront qu'il s'agit là d'une bien étrange façon d'obtenir sa rédemption ! C'est en fait dans cette confrontation avec les humains que le damné peut voir sa peine s'achever. Il existe plusieurs possibilités d'éliminer, et donc de sauver, un damné. La plus fréquente est sa crémation ; feu qui rappelle celui du purgatoire. Il y a aussi l'affranchissement du damné par la 
célébration de messes à son intention, comme le montre cet autre extrait d'un récit de damné :

[...] Comme lui avait expliqué [Dieu, Malikacha] le frappa à la tête avec une grande aiguille et le damné s'écroula. Eh oui il mourut ! Alors il dit [à Malikacha] :

- Comme tu m'as sauvé tu vas aussi sauver ma mère. Tu feras dire une messe pour nous. Il y a une pierre sur un monticule de cendre, c'est là qu'est enterré notre argent. Nous avons une paire de taureaux. Tu les vendras et avec cet argent tu feras célébrer une messe.

Puis, il s'envola sous la forme d'une colombe.

[...] Hinaspa aqnata nispa sirtuta nisqanta hina takarqun ankhay umanta yawriwan takarqum. Chaysi k'aq nispa kukuchiqa chutarqakapun $i$, ari wañurqapun. Chaysi nin :

- Ay ñataq nuqatas salvawanki hinas chaypachaqa entonces mamitaytawansi salvawanki.

Hinaspataqmi ichaqa nuqaykuta misakuyta ruwachiwankiku. Kachkanmi aqna rumin uspha muqupatapi, anchaypi chaypin qulqiyku p'ampasqa kachkan nispas nin i, turuykun kachkan, masa turu. Anchayta bindispa anchay qulqiwan misata ruwachiwankiku nispas nin.

Chaysi paloma phar phalaripun. (Luciana Poccohuanca, communauté de Pampallacta, Calca, 16/12/1997)

Cette « libération » du damné, obtenue grâce à l'action humaine, permet au mort de partir du monde des vivants et d'atteindre finalement le paradis. Sa transformation en mort non-prédateur est rendue possible après l'élimination de ses péchés, visible lors de sa transformation en colombe. La délivrance des damnés constitue une différence notable avec la doctrine catholique offricielle, qui voue ces âmes aux feux éternels de l'enfer sans qu'ils ne puissent jamais espérer en sortir. Par leur identité même, les damnés ne sauraient, selon cette même doctrine, bénéficier de l'aide (suffrages ou messes) de leurs familles : celle-ci s'avèrerait inutile car leur salut est impossible. Cependant, si les théologiens ont uniquement réservé au purgatoire les auteurs des péchés véniels, des visionnaires ont aflirmé que le rachat des péchés capitaux était possible (Carozzi 1994, p. 638). À l'époque coloniale, les missionnaires catholiques avaient largement développé au Pérou l'arme redoutable de la damnation des païens et des idolâtres et les avaient menacés du séjour définitif en enfer où ils subiraient d'atroces souffrances (Duviols 1971). D’ailleurs, le développement de l'iconographie infernale dans les Andes durera jusqu'au XIX ${ }^{\mathrm{e}}$ siècle, alors qu'en Europe, à la même époque, le thème de l'enfer n'était plus l'objet d'une telle imagerie (Estenssoro 2003, p. 288). L'effrayante fresque murale sur l'enfer de l'église de Huaro (province de Quispicanchis, Cuzco), peinte au début du XIX ${ }^{\mathrm{e}}$ siècle, en est une illustration (Figure 5). Pourtant, si les récits sur les damnés commencent effectivement par affirmer qu'ils ne pourront jamais être arrachés à leurs tourments, qualifiés d' "éternels ", ces morts finissent par gagner leur salut. Rappelons que c'est à l'époque coloniale que l'expression wiñay wiñay fut établie comme traduction quechua du concept 


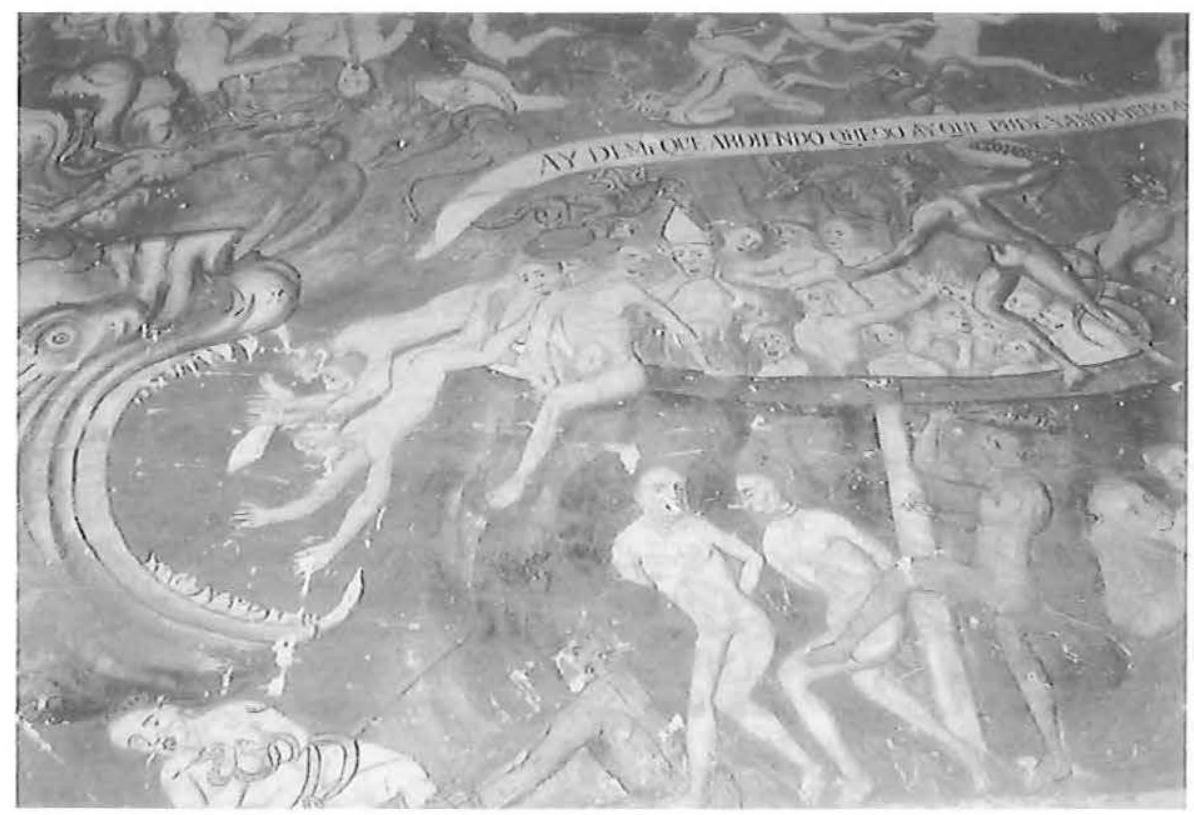

Fig. 5 - Détail de l'enfer, peinture murale de l'église de Huaro, département de Cuzco, Pérou, par Tadeo Escalante, début XIX ${ }^{e}$ siècle (cliché Robin)

d'« éternité », nouveau dans les Andes (César Itier com. pers.). Mais il semble que cette notion n'a pas été intégrée avec la signification que les évangélisateurs avaient souhaité inculquer aux Indiens, ainsi que le précise notamment le sermon II du Tercero Cathecismo de 1585 pour expliquer le sort des âmes pécheresses qui se damnent en enfer pour l'éternité ${ }^{32}$. En effet, wiñay wiñay indique aujourd'hui une période certes très longue, mais qui n'est pas forcément sans fin. Cette expression n'a pas le sens irrévocable d'éternité dans l'acceptation chrétienne lorsqu'elle se réfère au séjour des morts dans l'ukhupacha, en enfer. Un synonyme de cette formule est watan watan qui signifie « de nombreuses années ». Ainsi, l'éternité des souffrances endurées par les damnés a bien une fin. Les damnés réussissent, eux aussi, à rejoindre le reste de la communauté des morts, au paradis, au hanaqpacha. L'ukhupacha ne peut donc pas être considéré comme leur destination ultime.

Il ne fait nul doute que la montagne et/ou le volcan ne sont pas les résidences définitives des morts, ad mortem aeternam, ni même de certains d'entre eux. C'est bien plutôt le concept de purgatoire infernalisé qui a fini par être intégré. Doit-on alors parler d'échec de la politique évangélisatrice, lorsque l'on voit que l'enfer n'a pas été intériorisé comme lieu de damnation définitive des âmes ? Ou s'agit-il, 
au contraire, d'un succès missionnaire ? Par l'intermédiaire du purgatoire, en tout cas, c'est un contrôle extraordinaire sur les fidèles que l'Église catholique a mis en place. La célébration de la Toussaint semble bien le confirmer.

\section{LE RETOUR BIENVEILLANT DES MORTS OU L'ÉCHANGE POSSIBLE AVEC LES VIVANTS}

La célébration de la Toussaint (Todos Santos) est un événement central de la vie sociale des communautés paysannes et des villages des Andes de Cuzco. La première Toussaint qui suit un décès prolonge et finalise les funérailles du mort. Elle reproduit exactement leur structure rituelle, sur un laps de temps plus court. Cette commémoration du mort scelle la fin de son voyage vers l'autre vie et son entrée au pays des morts, au paradis, puisque c'est Dieu qui l'envoie temporairement sur terre : « À la Toussaint, Dieu envoie sur terre tous les morts du paradis. C'est pourquoi ils descendent tous ici pour le jour des morts. [Dieu] les envoie vingt-quatre heures, pour une seule journée ${ }^{33}$.

\section{Les intermédiaires privilégiés avec les morts : le prêtre et le diseur de prières}

Pour établir la communication avec les morts et les faire revenir à cette époque de l'année, certaines personnes servent d'intermédiaires privilégiés : les prêtres et les diseurs de prières. Le rôle des premiers apparaît clairement à l'occasion de l'office célébré le $1^{\text {er }}$ novembre. À la différence de la messe funèbre censée aider le mort à quitter le monde des vivants (messe d'enterrement ou « des huit jours »), celle de la Toussaint l'aide à revenir sur terre. Généralement, aucune messe n'est célébrée dans les communautés paysannes, faute de prêtres, voire de chapelles. C'est le cas à Lutto, Chumbivilcas. En revanche, une messe en l'honneur des défunts est célébrée le $1^{\mathrm{er}}$ novembre à Santo Tomás (capitale de Chumbivilcas). De nombreux habitants des communautés environnantes, qui ont un mort enterré dans le village, s'y rendent. Revenons sur l'épisode de l'oflice célébré le $1^{\text {er }}$ novembre 1998. L'église était comble. Des bouteilles d'eau avaient envahi le devant de l'autel pour être bénies par le prêtre avant d'être emportées au cimetière. Les bancs et les allées étaient surpeuplés, jusqu'à la porte où l'on se bousculait afin d'assister à la messe. Avant de commencer; le prêtre avait fait passer une liste sur laquelle ceux qui le désiraient pouvaient inscrire le nom du défunt qu'ils venaient célébrer en ce jour. Une foule de personnes s'empressèrent d'y faire figurer leur mort. Au bout du compte, cette liste contenait une centaine de noms. Après avoir commencé à la lire, le prêtre voulut écourter l'énumération des noms, car la cérémonie avait déjà duré très longtemps et l'église était plongée dans un brouhaha incessant. Si un désordre sonore semblait déjà régner, cette déclaration fit retentir une clameur générale de désapprobation. En effet, chacun souhaitait que le nom de son défunt fût clamé haut et fort par le prêtre. Le Père 
Pablo précisa qu'il avait bien pris note de tous les morts inscrits sur le papier et que la messe était achevée. Mais le tapage et les voix continuaient à s'élever. Malgré la longueur de la liste, le prêtre dut donc se plier aux exigences de ses ouailles, contre son gré. Leur insistance lui semblait une ineptie d'autant plus saugrenue qu'il était parallèlement convaincu que personne n'écoutait ses prêches. Ce qui n'était pas totalement faux. Cependant, l'insistance avec laquelle le public exigea d'entendre le prêtre prononcer le nom de chaque défunt n'est pas dénuée d'intérêt. En effet, cela témoigne de l'efficacité symbolique qui est accordée à la parole du prêtre. Sa récitation des noms est performative en soi. Mentionner le nom du défunt revient à établir une liaison plus directe avec son âme. Le prêtre est censé communiquer avec les morts plus facilement que n'importe quel être humain, les appeler à revenir dans le monde des vivants : "[Les âmes arrivent] au milieu de la messe, [quand] le prêtre célèbre l'office » ${ }^{34}$. Sa parole et sa présence même assurent, avec efficacité, la venue des morts sur terre. Ce rôle de médiation avec les morts ${ }^{35}$ est plus perceptible encore dans le cimetière avec les diseurs de prières.

D'ordinaire, le cimetière est évité et laissé à l'abandon. En revanche, pour la fête des morts, il redevient un lieu socialisé et très fréquenté. Ceux qui ont un mort dont on célèbre la première Toussaint s'adonnent à des préparatifs particulièrement élaborés. Dans le village de Santo Tomás, comme dans la communauté de Lutto, les gens affluent dans le cimetière le $1^{\mathrm{er}}$ novembre dès le lever du soleil. En fin de matinée, le cimetière est bondé. Chaque famille étale des étoffes tissées, près de la sépulture de leur défunt, sur lesquelles des offrandes de nourriture sont déposées à son intention. La présence physique du « retour » de l'âme du mort se manifeste lorsqu'un insecte volant s'approche des offrandes; certains versent de l'eau bénite dans un récipient afin d'attirer l'insecte. Puis, la famille s'assoit, attendant le passage d'un des nombreux diseurs de prières qui parcourent le cimetière pour offrir leurs services. En rétribution des prières récitées, le diseur de prières prélève les offrandes et devient le médiateur indispensable entre les vivants et leurs morts ; ses oraisons aidant à faire revenir l'âme du défunt parmi les siens. Les diseurs de prières sont généralement les principaux intermédiaires que l'on trouve à la Toussaint, mais ce ne sont pas les seuls personnages à assumer ce rôle. Le prêtre possède la même fonction d'intercesseur avec les âmes des morts. Il est même considéré comme plus efficace dans la communication avec les défunts dans la mesure où il jouit d'un statut hiérarchique supérieur à celui du diseur de prière qui est, pourrait-on dire, une sorte de prêtre au rabais. À Santo Tomás, lorsque le Père Pablo s'est rendu au cimetière après l'office, il a été sollicité afin de prier auprès de certaines tombes. Son refus systématique de récupérer les aliments servant d'offrandes a surpris les gens. Cela a créé un quiproquo entre cet homme qui « refuse de déposséder ces pauvres gens » et les personnes qui attendent de lui qu'il les emporte. Par le biais de celui qui prie, le mort est censé être nourri et honoré. Lorsque celui qui prie achève ses oraisons, il doit nécessairement retirer 
les offrandes. La famille les remplace immédiatement par une nouvelle série d'offrandes permettant de réciter à nouveau d'autres prières. La répétition de ces actes, qui peut durer des heures, permet d'assurer l'efficacité du rituel du retour des morts. On comprend donc que l'absence de collaboration du curé empêchait ce cycle de se reproduire normalement. Ce prêtre nord-américain qui refusait les offrandes considérait en fait ces « coutumes » comme le fruit de superstitions à peine acceptables.

Pourtant, ces gestes rituels relèvent-ils de pratiques véritablement hérétiques, restées incontrôlées par l'Église, et constituent-ils une preuve supplémentaire de la persistance de rites préhispaniques ${ }^{36}$, comme certains l'affïrment ? En se penchant à nouveau sur le sermon XXX du Tercero Cathecismo (Tercer Concilio Limense 1867, pp. 372-373), on trouve un passage très intéressant sur le bon usage des offrandes de nourriture faites à l'occasion des funérailles (avec mention du lama). Ce texte indique clairement que les offrandes pour le mort se perpétuent à l'époque coloniale. Celles-ci ne sont pas seulement tolérées, elles sont encouragées. Bien évidemment, il est précisé que les Indiens ne doivent aucunement croire que ces aliments sont destinés à nourrir les trépassés, sottise, précise le sermon. Ces aumônes servent au salut des âmes du purgatoire, raison pour laquelle, avant d'être reçues par le Christ, elles doivent être remises aux prêtres. Le signifiant est maintenu pour en modifier d'autant mieux le signifié. La nourriture ne doit plus être destinée à alimenter les défunts mais à être transférée à Dieu. On comprend dans ces conditions que les prêtres soient devenus les intermédiaires autorisés et quasi exclusifs entre les Indiens et leurs morts. Quel contrôle formidable sur leurs fidèles s'offrait ainsi aux ecclésiastiques, qui entraient au cœur de la relation avec les défunts ! Le pouvoir du prêtre augmente, de même que l'encadrement des fidèles. Dans la mesure où le culte des ancêtres constituait une source de préoccupation importante pour l'Église, n'était-il d'ailleurs pas logique de la voir s'immiscer dans les rapports aux ancêtres et chercher à investir l'imaginaire de la mort ? Ce rôle d'intercesseur du curé ou de son équivalent de rang inférieur, le diseur de prières, est, on l'a vu, toujours d'actualité dans les Andes. Ce qui n'empêche pas certains d'entre eux de voir dans les manifestations actuelles de la relation aux défunts des restes d'un ancien paganisme !

\section{L'opulence du paradis et la fertilité associée aux morts}

Revenons sur l'alimentation du mort et l'obligation que les hommes ont de le nourrir à son retour. Cette idée se trouve explicitée dans ce commentaire :

[Les morts] repartent en emportant les offrandes de nourriture. Ils ne mangent que ça durant l'année [suivante]. Si les gens ne leur ont rien laissé, ils repartent en s'aidant les uns les autres, tristement. C'est pourquoi Dieu leur laisse de la nourriture déjà prête au paradis. La nourriture n'est pas très bonne, mais les morts passent leur temps à en manger. 
Chayqa of rendata mikhunata aparikuspa ripun. Chayllatas watantin mikhukun. Mana churanku chayqa q'alallas ripun "mana nuqaqqa imallaypas kanchu. Mana kasqachu imallaypas» nispa. Hukta yanaparikuspalla khuyayta ripun. Taytanchissi churan chaypaqqa mikhunata listota Hanaqguluriapiqa chay. Mana mikhuna valinchu chayta mikhuspalla kakun riki. (Julián Poccohuanca Apaza, communauté de Pampallacta, Calca, 22/01/1999)

L'indication sur les aliments abondants au paradis, mais non nourrissants, semble justifier le rôle que les vivants doivent toujours jouer à l'égard de leurs morts, même lorsque ces derniers ont obtenu leur salut. Elle souligne l'interdépendance qui les unit. Les hommes espèrent en retour l'aide de leurs morts qu'ils implorent, justement dans le cadre de la production agro-pastorale, activité principale des communautés andines ; leurs demandes concernent en particulier la venue des pluies, les récoltes, la multiplication du bétail, voire la protection contre les voleurs. Cette fonction attribuée aux morts doit être rapprochée du caractère fécond qui est attaché au paradis dans lequel ils vivent ${ }^{37}$. Si ce lieu se définit par son inversion par rapport à la vie terrestre (ceux qui sont malades ici-bas seront guéris dans l'autre vie), c'est également l'endroit par excellence de l'opulence et de la fertilité. La nourriture y foisonne. Elle ne manque jamais et se multiplie automatiquement dès qu'elle est consommée par les morts. Ainsi, le retour des morts sur terre est censé fournir aux vivants une partie des biens dont regorge le paradis d'où ils viennent. Pour cela, il est nécessaire de commémorer correctement ses morts récents. Dans le cas contraire, l'âme pourrait repartir insatisfaite et frapper d'infortune (ruine de récoltes, sécheresse, etc.) la communauté toute entière, comme le signale Gabriela Vera :

Si les gens ne mettent pas des offrandes à temps, [le mort] repart en pleurant. Il se fâche et envoie la famine à ses enfants et à ses brus. On doit se comporter [avec le mort] de la même manière qu'avec nos amis vivants et il faut lui préparer de bonnes choses. Avec ça, on dit que [Dieu] ne peut refuser [d'aider les hommes]. Il pourvoit toujours [à nos besoins] et donne beaucoup d'argent et de nourriture à ses enfants. Si tu ne t'occupais pas [des morts], tu vivrais dans la tristesse et la pauvreté.

Sis mana churapunku[chu] listuta chayqa waqaspas kutipun. Phiñakunsiyá " manaña wasiypiqa tarikusqayñachu imaypas hayk'aypas " nispa. Aswayta yarqaytas saqiyatamun chay wawankunaman qhachunninkunamanpas. Anchaysiyá hina kawsaq masinchisman hina sumaq tranquilo churana suyachinayku. Anchaywanqa manas neganmanchu. Churallansi. Asway allinta wawanmanhina qumushan Diusninchisyá qulqitapas mikhunatapas qumushan. [...] Mana aqnata kasurpariwaq chayqa manas usachikuwaqchu ni medio hukraltapas. Khuyaysi pobre puriwaq. (Gabriela Vera, annexe de Ch'alla Ch'alla, communauté paysanne de Lutto, Chumbivilcas, 26/06/2000)

La nécessité de célébrer convenablement ses morts a pour but de bénéficier des bienfaits octroyés, en fait, par Dieu lui-même. En leur faisant des offrandes, les hommes peuvent espérer en retour que Dieu augmentera les ressources des 
vivants. Par ailleurs, la pluie doit commencer à tomber à partir de la date de la Toussaint qui clôt rituellement la saison sèche. Le rôle des morts dans la venue de cet élément vital est manifeste à travers la figure des angelitos (enfants baptisés et morts en bas âge), censés apporter l'eau sur terre. Certaines versions rapportent que ces petits anges filtrent l'eau sale qui sort de la montagne pour l'amener aux hommes, nettoyée et limpide. C'est pourquoi à Pampallacta de petites cruches en céramique sont déposées sur les tombes des enfants afin qu'ils envoient de l'eau à leurs parents.

Le 2 novembre, le séjour des morts parmi les vivants prend fin. Ils doivent repartir vers leur pays, au paradis, emportant avec eux les requêtes formulées par les vivants. Ils repartent une fois que le diseur de prières est passé récupérer les offrandes, une disparition qui rappelle celle, par crémation, des dons funéraires.

\section{Conclusion}

L'analyse des récits eschatologiques individuels a mis en évidence l'influence fondamentale exercée par l'Église coloniale sur l'imaginaire de la mort. Les références à certains passages des Écritures, probablement diffusés à travers les récits édifiants des exempla, sont évidentes. Mais c'est surtout dans les conceptions relatives au purgatoire que l'intervention missionnaire est la plus manifeste. Dans le contexte post-tridentin de la Contre-Réforme, l'enseignement du purgatoire et le développement des rites dédiés aux âmes du purgatoire ont joué un rôle primordial au Pérou. Dans sa lutte contre le culte préhispanique des ancêtres, l'Église catholique a su exercer un contrôle extraordinaire sur les nouveaux convertis par l'encadrement de la relation qui unissait les Indiens à leurs défunts. On en trouve notamment la trace dans la médiation entre les vivants et leurs morts, telle qu'elle est pratiquée par les prêtres aujourd'hui. Étonnamment, hormis les récents travaux d'Estenssoro (2003), peu d'attention a été portée jusqu'ici au purgatoire qui, on l'a vu, occupe pourtant une place centrale dans le périple posthume de l'âme. Bien que le nom purgatorio, diffusé par l'Église, ait connu une postérité mitigée - son emploi étant désuet de nos jours -, le concept qui lui est associé jouit d'une pérennité remarquable, évidente dans les réélaborations actuelles des représentations de l'au-delà. Certes, les termes utilisés pour s'y référer rappellent un " enfer » (infierno, supaywasi, ukhupacha...). Mais c'est précisément un purgatoire infernalisé que l'iconographie et les textes religieux coloniaux évoquent à satiété. Il a fini par s'imposer autour de trois éléments : un lieu, la montagne-volcan; un procédé, le feu purificateur qu'elle contient ; une durée, le séjour temporellement limité en son sein.

On a aussi constaté que c'est dans une économie du salut que la relation des hommes à leurs défunts peut être éclairée. En effet, le caractère pathogène attribué à ces derniers au temps des funérailles n'est véritablement compréhensible, 
aujourd'hui, que s'il est mis en rapport avec les péchés qui caractérisent les êtres humains au moment du décès (à l'exception des enfants baptisés, les innocents angelitos). La pénitence endurée lors du voyage purgatoire vers l'autre vie permet la destruction de cet "homo peccator », pour reprendre l'expression de Danièle Dehouve (2004, p. 15), qui définit le mort avant qu'il ne soit sauvé. Par la suite, le retour temporaire des morts ne présente plus un risque de prédation. Bien au contraire, l'obtention du salut et l'intégration au paradis (hanaqpacha) ouvrent le début d'un cycle d'échanges entre les vivants et leurs défunts. Celui-ci est ritualisé durant la première célébration de la Toussaint après le trépas, célébration qui clôt les funérailles. C'est donc une relation triangulaire qui s'établit : les morts sont les intercesseurs entre Dieu et les hommes, médiateurs de la substance divine, source de fertilité agro-pastorale. Ainsi, le binôme péché/salut fait pendant au couple prédation/échange.

On est donc bien loin de l'idée d'ukhupacha, terme employé par l'anthropologie et l'ethnohistoire andine, en référence à la "conception indigène » d'un " monde du bas », inframonde aux pouvoirs génésiques, peuplé de personnages chtoniens, héritiers d'anciennes divinités préhispaniques satanisées par les missionnaires ${ }^{38}$. Les récits et les gloses, recueillis dans le département de Cuzco, ne permettent pas d'aller dans un tel sens, puisque ukhupacha renvoie systématiquement aux notions chrétiennes d'enfer et de purgatoire et à elles seules. Ce terme est quasi exclusivement employé de nos jours pour désigner ce lieu de passage des âmes vers l'autre vie. Par ailleurs, si l'on retrouve effectivement un lien entre les morts et la fertilité, c'est bien au contraire dans leur localisation dans le hamaqpacha qu'il apparaît, non dans l'ukhupacha, séjour temporaire des morts et damnés, qui serait plutôt un emplacement associé à la stérilité. Ce commentaire final ne signifie nullement que le monde souterrain ne soit pas, lui aussi, lié à l'abondance et à la fertilité. Il suffit de mentionner la Pachamama, la Terre-mère qui octroie la fertilité agricole, ou encore les diables des mines, pourvoyeurs de filons (Absi 2003 ; Salazar 2002). Mais les morts ne sont pas associés à ce type de fertilité tout comme les entrailles de la terre ne relèvent pas nécessairement d'un ukhupacha. Si certains récits font apparaître le diable dans l'ukhupacha, c'est toujours en relation avec les récits eschatologiques individuels. Aucune interaction n'est alors envisagée avec eux. Ces diables apparaissent uniquement comme des personnages stéréotypés, infligeant des châtiments à l'âme dans son voyage posthume. L'application du concept d'ukhupacha à un inframonde andin, source de fertilité, semble donc inappropriée. Il ne faut pas confondre l'étymologie de ce terme, qui évoque un espace souterrain ${ }^{39}$, et les conceptions qu'il véhicule aujourd'hui. Le « monde du bas » n'est jamais qualifié d'ukhupacha par les membres des communautés de Cuzco, puisque les représentations qui lui sont liées sont, on l'a constaté, radicalement différentes de celles d'un inframonde fertile. *

* Manuscrit reçu en février 2004, accepté pour publication en mai 2004. 


\section{NOTES}

Remerciements : à Pierre Duviols pour ses conseils toujours avisés, ainsi qu'à Juan Carlos Estenssoro Fuchs pour ses généreuses relectures.

1. Les données présentées proviennent des communautés de Pampallacta et de Lutto où j'ai effectué mes enquêtes ethnographiques entre 1995 et 2002. Les récits mentionnés dans le texte, retranscrits en quechua et traduits en français par moi-même (V. R.), ont été enregistrés au cours de ces différents séjours. Ils ont été retranscrits selon les normes de l'écriture normalisée du quechua, dans sa variante dialectale cuzquénienne.

2. Les termes employés pour se référer à l'âme humaine sont alma et animu, utilisés en quechua de façon interchangeable. L'origine de leur emploi reste obscure, puisque seul le terme latin anima apparaît dans les textes de catéchèse coloniaux en quechua (César Itier com. pers.). L'utilisation générique d'alma pour qualifier le défunt s'explique probablement par l'ambiguïté, en espagnol même, de ce mot : alma désigne l'âme, le principe vital immatériel de l'individu, et le mort, dans sa dimension matérielle de cadavre. L’âme est " renvoyée » lors du rite de clôture des funérailles, mais les péripéties qu'elle connaît dans l'au-delà font l'objet d'abondants commentaires qui retiendront toute notre attention. Pour une étude plus détaillée des conceptions du corps et de l'âme, voir Robin 2002, partie I, chap. I " corps et âmes : les conceptions de la personne ».

3. La mise à mort rituelle doit nécessairement se faire par strangulation. Si jamais son sang se répand, le chien n'est plus considéré comme étant en mesure d'aider l'âme dans l'autre vie. On retrouve ici l'idée du sang comme vecteur de force vitale et de son écoulement comme synonyme de souillure. Le sacrifice sanglant du chien serait alors jugé inutile, voire contre-productif.

4. Pour plus d'informations sur les rituels funéraires de la région, voir Robin (2002, pp. 48-78; 2003).

5. Notons que l'on trouve également la mention d'un autre Lazare biblique : Saint Lazare. En effet, le fleuve traversé par le mort à la sortie du village des chiens est parfois désigné comme le "fleuve Saint Lazare » (San Lazaro mayu). Saint Lazare est évidemment un tout autre personnage que le pauvre Lazare mentionné dans l'Évangile selon Saint Luc. Toutefois, il semble s'être produit, dans la tradition orale, une fusion d'identité entre le pauvre Lazare et le Lazare ressuscité par le Christ. Cet amalgame peut s'expliquer. Il est probablement lié au fait que le passage de l'Évangile selon Saint Jean (St Jn $11-1 / 44$ ) sur la résurrection de Lazare, lui aussi bien connu dans le département de Cuzco, est également lu à l'occasion des messes de funérailles et de la Toussaint. Par ailleurs, ce passage de l'Évangile a été amplement propagé dans les Andes depuis le Xvil siècle, comme l'atteste par exemple le cantique V (espagnol/quechua) du Symbolo Catholico Indiano du franciscain Luis Jerónimo de Oré, édité en 1598. Il fut officiellement adopté dans l'évêché de Cuzco à partir de 1601 et les cantiques devaient être chantés au quotidien dans les paroisses d'Indiens (Pello s.d.). Notons également le sermon sur la résurrection de Lazare par le Christ, établi en espagnol et traduit en quechua par le franciscain Diego de Molina en 1649 (Taylor 2002, pp. 187-212).

6. On citera le témoignage du cultivateur Arnold Buschmann, dans le diocèse de Cologne au $\mathrm{xv}^{\mathrm{e}}$ siècle, qui eut la vision de l'âme en peine de son grand-père, décédé des décennies auparavant. Ce dernier lui apparut pour implorer son aide afin de gagner son salut. Il le renseigna alors sur les moyens dont disposent les hommes pour apaiser les souffrances purgatoires endurées par les âmes pécheresses, comparant leur sort à celui du mauvais riche (Lecouteux 1999, p. 75).

7. Le terme de misti, tel qu'il est employé par les paysans des communautés, se réfère à la population qui vit en dehors desdites communautés, qu'il s'agisse des propriétaires terriens des haciendas avant la réforme agraire de 1969 ou, de façon générale, de toute personne habitant dans les villages de la région : commerçants, autorités politiques, etc., avec lesquels les membres des communautés tissent des rapports qui se caractérisent souvent par la dépendance. Garder le terme quechua de misti dans le texte est un choix. Le but est d'éviter l'emploi de "métis » (traduction du mot liée à son 
origine espagnole " mestizo »). " Métis » est en effet parfois utilisé, au Pérou, dans une acception un peu floue qui oblitère le caractère presque exclusivement social, et pas tant biologique, du terme " misti », au risque de tomber dans une réification des « identités ». On peut en effet être un paysan de communauté à sa naissance pour devenir, à la suite de migrations et au terme d'une ascension socio-économique, un misti, à travers un processus que l'on qualifiera de misti-fication.

8. " mistis wiraquchas chaykuna señoras mana chaykuna q'ipinchu " (Julián Poccohuanca, communauté de Pampallacta, Calca 16/11/2000).

9. "' 'mistin kani kay camisayta qhillicharukuyman, mana q'ipiykimanchu' nispa nin chay dueñonta " (Pablo Molina, annexe de Ch'alla Ch'alla, communauté de Lutto, Chumbivilcas, 20/03/1999).

10. C'est ce qu'explique Gabriela Vera lorsqu'elle se réfère à ces lieux : "Vivant, on ne peut y arriver. Ce n'est que mort qu'on peut y arriver » ("Kawsaqqa chayanmanchu manan riki. Wañuqllas chayasumman », annexe de Ch'alla Ch'alla, communauté de Lutto, Chumbivilcas, 20/03/1999).

11. "Volcan. Pero mana chayanichu. Imayá chaypi? Pero volcan chaymanpuni risunqa wañuspaqa nispapuni nin. Volcanmanpumi chayasummanqa volcanman Qurpunaman" (Pablo Alvis, annexe de Ch'alla Ch'alla, communauté de Lutto, Chumbivilcas, 26/06/2000).

12. "Huk vidapi riki imaynas riki huk vidapi. Volcanta pasanraqchá riki. Arequipa volcanchá. Salqantaychus kanman Pichupichu? " (Apolinaria Molina, annexe de Ch'alla Ch'alla, communauté de Lutto, Chumbivilcas, 23/07/2000).

13. " Dès que [l'âme] arrive au volcan elle va d'abord y brûler " ("Volcanmanhina chayan chaypiraqchá rawran », Pablo Molina, annexe de Ch'alla Ch'alla, communauté de Lutto, Chumbivilcas, 23/07/2000).

14. Le texte qui figure entre double crochets [[...]] est extrait d'une autre discussion sur le même sujet que j'ai eue avec Pablo Molina le 23/07/2000. J'ai réinséré ce passage dans V1 afin de donner d'autres éléments de description de ce lieu, sans toutefois reproduire l'intégralité de la variante racontée par Pablo Molina.

15. Notons au passage l'analogie entre la douleur expérimentée par les âmes et les souffrances vécues par les malades qui ne guérissent pas malgré des séjours prolongés à l'hôpital. Cette comparaison est révélatrice du peu de confiance à l'égard des institutions hospitalières. Les paysans séjournent rarement dans les hôpitaux et s'y rendent avec réticence. Les vexations infligées par le personnel soignant, que j'ai observées en accompagnant des amis de la communauté de Pampallacta à l'hôpital régional de Cuzco, représentent probablement un des motifs de résistance. Mais c'est l'ineflicacité des traitements médicaux subis qui est évoquée et s'avère malheureusement souvent confirmée. Ces raisons expliquent la réserve qu'ont souvent les paysans à l'égard des médecins et des décisions prises, parfois tardivement, de les consulter.

16. "Maypachan huchan tukun chayasqa [...] q'usñishaspallas yassssta wata watan. Huchanmanta q'usñishan [...] chay tukukun chayasqa chay punkumanta yuraq paloma phalarikapun » (Julián Poccohuanca, communauté de Pampallacta, Calca, 16/11/2000).

17. "Kawsarinchá Diospa munayninpi riki kanasqapas. Hinaspa ripun riki alma llaqtaman »(Pablo Alvis, annexe de Ch'alla Ch'alla, communauté de Lutto, Chumbivilcas, 26/06/2000).

18. "Chayqa imayna condicionpiñas riki alma librakuspa hanapachata siqayapun Taytachaman. Chaypiqa riki rawrayta tukuqtinqa... huchanmanta chaypi rawran. Anchaymanta pampachakunchá imaynachá. Chaymantaña librakum » (Pablo Molina, annexe de Ch'alla Ch'alla, communauté de Lutto, Chumbivilcas, 23/07/2000).

19. Gabriela hésite et ne finit pas sa phrase mais voulait probablement indiquer que Dieu nous juge selon le type et la quantité de nos péchés.

20. Voir par exemple ce passage du texte de Valderrama et Escalante (1980, p. 233), devenu une référence pour qui s'intéresse au voyage du mort vers l'au-delà : " tuvimos ocasión de presenciar y hacer el seguimiento de ritos y ceremonias de entierro semejantes a aquellas narradas por los cronistas y extirpadores de idolatrias del siglo xv y que, con algunas variaciones o sincretismos se siguen practicando en la actualidad; demostrando una vez más la capacidad de lucha y de resistencia de la cultura y del pueblo andino ». 
21. Régions qui recouvrent une partie des départements actuels de Cuzco - dont la province de Chumbivilcas -, d'Apurimac, d'Arequipa et de Puno.

22. Certains passages des récits eschatologiques présentés par Valderrama et Escalante (1980) mentionnent Dieu ou le paradis. Toutefois, ces éléments, minimisés lorsqu’ils sont évoqués, sont rapidement évacués de la préoccupation des auteurs. Rosell (1976) s'attarde davantage sur la composante catholique du voyage des morts mais pour insister ensuite sur la signification essentiellement préhispanique de ces récits.

23. À titre d'illustration, l'introduction écrite par Flores Ochoa pour le livre Q'ero, el último ayllu inka (Flores Ochoa et Núñez del Prado 1984, p. x.) est tout à fait révélatrice de ce double terrain sur lequel se placent ces anthropologues engagés dans un projet politique régionaliste : « On ne peut nier que l'anthropologie cuzquénienne a eu une forte tendance indigéniste, qu'elle conserve encore et dont témoignent les anthropologues actuels les plus lucides. [...] nous disons indigéniste(s) dans son sens originel et authentique, qui implique une attitude d'engagement, de défense de l'“andinité". [...] C'est l'anthropologie qui a hérité de l'indigénisme, l'esprit hautement révolutionnaire de cette époque, puisqu'il était fondé sur la recherche du changement inspiré par l'“andinité", du Tawantinsuyu [empire des Incas] comme modèle pour construire la société du futur et des symboles andins comme références idéologiques et d'action » [traduction de l'auteur, V. R.].

24. Il me semble que cette idée est similaire à celle que l'on trouve avec le "mythe d'Inkarri ". Estenssoro (2003, pp. 355-356) a trouvé une origine possible de ce récit sur la résurrection de l'Inca - au corps démembré, réparti entre le Pérou et l'Espagne et qui finira par se rassembler pour renaître - dans un sermon du curé Francisco de Avila (1647-1648) qui évoquait la possible rédemption des ancêtres des indigènes. Le mythe d'Inkarri, "découvert » par les anthropologues dans les années 1950, a été interprété comme une forme populaire de résistance à la Conquête et comme l'exemple paradigmatique de " l'idéologie messianique du monde andin » (allusion à l'ouvrage Ideología mesiánica en el mundo andino édité par Juan Ossio en 1973). Estenssoro critique la manière dont les sciences sociales travaillant sur la figure de l'Inca ont usé et abusé du mythe d'Inkarri pour produire un discours où se confondent l'histoire et la volonté de définir une identité nationale à partir de l'existence de l'« utopie andine $"$.

25. Je reprends ici les termes de Flores Ochoa (Flores Ochoa et Núñez del Prado 1984, p. xv).

26. " muestra participación observante en estas ceremonias $[. .$.$] demuestra(n) la vigencia de cate-$ gorias mentales que constituian la antigua estructura del pensamiento andino » (Valderama et Escalante 1980, p. 241).

27. Pour ce rappel de la constitution du purgatoire, j'utilise et renvoie à l'ouvrage de référence $L a$ Naissance du purgatoire de Jacques Le Goff (1981, pp. 11-12 ; $24 ; 25 ; 243 ; 333-335,348$ ).

28. Sur la diffusion des exempla au Mexique, voir Dehouve $(2000 ; 2004)$ et, pour le Pérou, voir Estenssoro (2003).

29. Lieu appelé parfois « maison de feu » (nina wasi) comme pour l'« enfer » du volcan.

30. Voir, par exemple, Casaverde (1970, pp. 200, 203, 209).

31. " Mana nuqahina kankischu. Mamayta maqayukurani, chukchankunata hurquyırani. Ama huk timpu muqahina kankischu. Chaymantan Diosninchis qarqumuwan " (Benedicta Huillcayquipa, Santo Tomás, Chumbivilcas, 28/12/1998). Voir la transcription intégrale de ce récit dans Robin (2002, annexe 2).

32. « [...] Si queréis saber que cosa es el pecado, sabed que os hace esclavo del diablo, enemigo de Dios, condenado a los tormentos eternos del infierno, que el que muere en pecado va para sienpre jamás condenado al fuego, donde arderá sin fin y aunque más gritos dé con rabiosos dolores, nunca acabará de penar lo que merece por el pecado que hizo contra Dios » (Sermon II).

33. "Santospi q'alata hanaqpachamanta kachayamum Taytanchis. Chayqa q'ala huykumunku almakunaq p'unchayninpi kaypi. Vinticuatro horas kachay'amun huk p'unchayllapaq " (Julán Poccohuanca, communauté de Pampallacta, Calca, 22/01/1999).

34. "Chawpi misapisyá riki señor kura misashan " (Pablo Alvis, annexe de Ch’alla Challa, communauté paysanne de Lutto, Chumbivilcas, 28/06/2000). 
35. Cette fonction d'intermédiaire privilégié avec les morts n'est pas exclusive du cas andin, puisqu'on retrouve ce même rôle attribué aux prêtres du purgatoire dans l'économie funèbre en pays d'Oc, aux XIV et $\mathrm{XV}^{\mathrm{e}}$ siècles (Fournié 1987).

36. Un autre argument avancé par les tenants de la thèse de la survivance préhispanique est l'insecte comme incarnation du mort. La matérialisation du mort en insecte volant (généralement mouche ou papillon) est effectivement une représentation très ancienne dans les Andes, que l'on retrouve couramment à l'époque précolombienne. L'iconographie mochica montre le lien qui unissait les morts aux mouches (Hocquenguem 1981). Postérieurement, on retrouve cette représentation dans le premier texte colonial écrit en quechua sur les traditions préhispaniques (Taylor 1987). De même, les procès pour idolâtrie de Cajatambo mentionnent les mouches comme supports des âmes des morts (Duviols éd. 2003). Pour autant, il est difficile d'affirmer qu'il s'agit de survivance lorsque les concepts qui sont associés à ces représentations animales ont radicalement changé depuis les temps précolombiens.

37. Les termes hanaqpacha, hanaqgloria, hanaqpachagloria ainsi que gloria (la Gloire) et Jerusalem Ilacta (la Jérusalem céleste) sont indifféremment employés pour qualifier ce paradis dans lequel se trouve le pays des morts (alma llaqta).

38. Voir, par exemple, Bouysse-Cassagne et Harris (1987).

39. La construction de ce terme par l'Église coloniale s'est probablement faite à partir d'une métonymie (avec $u k h u$ "l'intérieur ou le dedans» et pacha "portion de temps ou d'espace»), permettant d'évoquer le monde infernal situé sous terre (Estenssoro 2003, p. 114).

\section{BIBLIOGRAPHIE}

Aвsı Pascale

2003 Les Ministres du diable, L'Harmattan, Paris.

Arringa Pablo Joseph

1999 Extirpación de la idolatría del Pirú, CBC, Cuzco [1621].

Avila Francisco de

1647-1648 Tratado de los evangelios que la iglesia propone todo el año, [s.n.], Lima.

BLoch Maurice et John ParRy, éds

1982 Death and the regeneration of life, Cambridge University Press, Cambridge.

Bourget Steve

1996 «Los raptores del alma : prácticas funerarias en la iconografía mochica », in L. Millones et M. Lemlij, éds, Al final del camino, SIDEA, Lima, pp. 40-60.

BouysSE-CASSAGNe Thérèse

1999 «Attention! Un diable peut toujours en cacher un autre: à propos de l'introduction des images de l'enfer chez les Indiens de l'altiplano bolivien », Traces, 34, pp. 22-39.

BouYsse-Cassagne Thérèse et Olivia Harris

1987 «Pacha : en torno al pensamiento aymara », in Tres reflexiones en torno al pensamiento andino, Hisbol, La Paz, pp. 11-60.

Casaverre Juvenal

1970 «El mundo sobrenatural en una comunidad », Allpanchis, 2, pp. 122-243.

CAROZZIClaude

1994 Le Voyage de l'âme dans l'au-delà d'après la littérature latine ( $\left.v^{e}-x u I^{e}\right)$, École française de Rome, Rome. 


\section{Dehouve Danièle}

2004 L'Évangélisation des Aztèques ou le pécheur universel, Maisonneuve \& Larose, Paris/université Paris X, Nanterre.

Dehouve Danièle, éd.

$2000 \quad$ Rudingero el borracho y otros exempla medievales en el México virreinal, CIESAS, Mexico.

Duviols Pierre

1971 La Lutte contre les religions autochtones dans le Pérou colonial. L'extirpation de l'idolâtrie de 1532 à 1560, Institut français d'études andines, Lima.

Duviols Pierre, éd.

2003 Procesos y visitas de idolatrías. Cajatambo Siglo XVII, Pontificia Universidad Católica del Perú/Instituto Francés de Estudios Andinos, Lima, coll. « Clásicos Peruanos ».

\section{Estenssoro Fuchs Juan Carlos}

2003 Del paganismo a la santidad, Instituto Francés de Estudios Andinos/ Instituto Riva Agüero, Lima.

Flores OchoA Jorge et Juan-Victor NúÑez Del Prado, éds,

1984 Q'ero el último ayllu inka, Centro de Estudios Andinos Cuzco, Cuzco.

FOURNIÉ Michelle

1987 "Les prêtres du purgatoire (XIv ${ }^{\mathrm{e}}-\mathrm{Xv}^{\mathrm{e}}$ siècles) », Études Rurales, 105-106, pp. 93-121.

1997 Le Ciel peut-il attendre ? Le culte des âmes du purgatoire dans le Midi de la France (1320 environ-1520 environ), Les éditions du Cerf, Paris.

\section{Galindo Pedro}

$1855 \quad$ Novena muy devota, en que se pide a Jesús crucificado, el alivio y consuelo de las benditas almas del purgatorio nuevamente reformada por un sacerdote devoto de aquellas santas almas y que desea establecer y aumentar más en los fieles tan provechosa devoción, [s.n.], Ayacucho.

Guaman Poma De Ayala Felipe

1987 Nueva crónica y buen gobierno, édité par R. Adorno, J. Murra et J. Urioste, Historia 16, Madrid [1613], coll. « Crónicas de América » 29.

\section{HARris Olivia}

1982 "The deads and the devils among the Bolivian Laymi ", in M. Bloch et J. Parry, éds, Death and the regeneration of life, Cambridge University Press, Cambridge, pp. 45-73.

\section{Hertz Robert}

1970 "Contribution à une étude collective de la représentation de la mort ", in Sociologie religieuse et folklore, PUF, Paris, pp. 1-83 [1907].

HocQuenguem Anne-Marie

1981 "Les mouches et les morts dans l'iconographie mochica », Ñawpa Pacha, 19 , pp. 63-69.

Jurado Palomino Bernardo

1943 "Declaracion copiosa das las quatro partes mas esenciales, y necesarias de la doctrina christiana », in Catechismus Quichuensis : ad fidem editionis Limen- 
sis anni MDCXLVI edidit, Latine vertit, analysi morphologica synopsi grammatica / indicibus auxit Hippolytus Galante, Hispanice e Latino reddidit Eliseus B. Viejo Otero, Consejo superior de investigaciones científicas, Madrid [1649].

Lecouteux Claude, éd.

1999 Dialogue avec un revenant, Presses universitaires de la Sorbonne, Paris.

Le Gorf Jacques

1981 La Naissance du purgatoire, Gallimard, Paris.

LOAYZa Leonor

1956 "Caminos y moradas de ultratumba », Archivos de Folkore peruano, 2, pp. 91-96.

Macera Pablo

1993 La Pintura mural andina, siglo XVI-XIX, Milla Batres, Lima.

Millones Luís et Moisés Lemlij, éds

1996 Al final del camino, SIDEA, Lima.

NúÑez Del Prado Juan Victor

1970 «El mundo sobrenatural de los quechuas del sur del Perú a través de la comunidad de Qotobamba ", Allpanchis Phuturinqa, 2, pp. 57-119.

ORÉ Luis Jerónimo de

1982 Symbolo católico indiano, Australis, Lima [1598].

Pello Xavier

s.d. «La langue au service de Dieu : les hymnes en quechua de Fray Luís Jerónimo de Oré (1554-1630) », Bulletin de l'IFEA, [à paraître].

2000 «Los últimos días de Luis Jerónimo de Oré (1554-1630) : un nuevo documento biográfico », Bulletin de l'IFEA, 29 (2), pp. 161-171.

RoBIN Valérie

2002 Miroirs de l'autre vie: rites et représentations des morts dans les Andes sud-péruviennes, thèse de doctorat en ethnologie, université Paris X, Nanterre.

2003 «Les chemins de l'autre vie ou les rituels funéraires dans les Andes sudpéruviennes ", Ateliers, 25, pp. 41-59, Laboratoire d'ethnologie et de sociologie comparative, Nanterre.

Rosell Victor

1976 El Entierro en Cotabambas, tesis de licenciatura en antropología, UNSAAC, Cuzco.

SAlazar-Soler Carmen

2002 Anthropologie des mineurs des Andes. Dans les entrailles de la terre, L'Harmattan, Paris.

Schmitr Jean-Claude

1993 Les Revenants, Gallimard, Paris.

TAYLOR Gérald, éd.

1987 Ritos y tradiciones de Huarochiri del siglo xVII, IEP, Lima. 
2002 Sermones y ejemplos, Biblioteca andina de bolsillo, IFEA, Lima.

\section{Tercer Concilio Limense}

1867 Tercero Cathecismo y exposición de la doctrina christiana por sermones, Librairie Rosa et Bouret, Paris [1585, Lima].

ValderRama Ricardo et Carmen Escalante

1980 "Apu Qorpuna : visión del mundo de los muertos en la comunidad de Awkimarka ", Debates de antropología, 5, pp. 233-264.

VOVELLE Michel

1996 Les Âmes du purgatoire ou le travail du deuil, Gallimard, Paris, coll. « Le temps des images $》$.

\section{ANNEXE}

\section{Du chien Lazare}

Julián Poccohuanca Apaza

(Communauté de Pampallacta, province de Calca, département de Cuzco, 11/01/1999)

Donc, il y avait un homme. Il avait un chien. Cet homme était devenu veuf mais n'était pas resté tout seul. Il possédait beaucoup d'animaux, beaucoup de moutons et beaucoup d'alpagas. [Un jour] il tua un mouton. Et le chien mangea la viande du mouton. Alors [cet homme] punit violemment le chien. Il le tua presque en lui disant : «bon sang, c'est toi qui as mangé la viande ". Alors le chien partit sur le volcan [ce détail signifie que le chien était comme mort car il a pris le chemin de l'autre vie]. La terre le fit revivre. Il n'était pas mort. Il partit sur le volcan. L'homme ne cessait de demander aux gens [qu'il rencontrait] : « où est passé mon chien? ». "Un chien vient effectivement de passer», lui disait-on. Il interrogeait constamment les personnes [qu'il rencontrait]. "Un chien vient effectivement de passer ", lui disait-on. Il partit sur les traces de son chien. Seul son petit chien, tel un homme, gardait tous [ses animaux] de nuit, et il les gardait aussi de jour. C'est comme ça que le petit chien faisait paître ses animaux. Et le jour, toujours très alerte, il faisait paître le bétail, les moutons et les alpagas en aboyant. Puis [l'homme] arriva auprès de Dieu. Il [Dieu] était là, [sous la forme d'] un monsieur. " Pourquoi es-tu venu ? », demanda-t-il. " J'avais un chien. Et il est venu jusqu'ici » [répondit l'homme à Dieu]. Alors il vit son chien qui était assis sur une couverture dans un coin. Celui-ci s'enfuit. "Là-bas, c'est mon chien ! ", dit l'homme. "Qu'est-ce que tu lui as donc fait ? », lui demanda [Dieu]. Il appela le chien. Et le chien, qui avait un nom, arriva. Dieu l'appela par son nom. Alors le chien arriva après que le seigneur l'ait appelé. Puis [l'homme] l'appela aussi par son nom en lui disant : " allez, on y va ». Mais [le chien] ne voulait pas revenir. Il ne voulait absolument pas. Finalement il n'est pas venu du tout. Et il dit [à son maître sur terre] : « c'est vrai j'ai mangé l'animal que je gardais. [Mais] nuit et jour j'ai gardé [ton bétail], même la nuit je l'ai gardé de tout danger, sans relâcher mon attention. Le jour je faisais paître le bétail, toujours en état d'alerte. J'ai mangé l'animal que je gardais et que je faisais paître, oui, mais est-ce pour ce petit mouton que tu te plains ? Quelle quantité [de bétail] un voleur aurait-il emporté ? Il en aurait sûrement emporté une grande quantité. Alors que moi je n’ai mangé 
qu'un petit bout de viande. Je ne m'en irai pas d'ici, va-t'en. Je ne m'en irai pas d'ici ». Et Dieu demanda [au chien ce qu'il voulait faire]. « Je ne m'en irai pas. Il m'a puni, Il m'a fait telle chose », répondit-il [à Dieu]. «Pourquoi m'as-tu puni ? Je suis Lazare, pourquoi m'as-tu puni, alors que je suis Lazare? », demanda [le chien à son ancien maître]. Alors [Dieu] appela un autre chien. C'était un grand chien, il était grand. [Dieu] lui dit en lui montrant l'homme : « mange, mange ça ». Le chien se leva [...] Il était grand et sa bouche était énorme comme ça. Alors le chien avala l'homme d'un seul coup, tout debout qu'il était [l'homme]. Puis il est parti en courant et le déféqua tel quel. Il déféqua l'homme tout debout qu'il était. De l'eau sortit, et cela balaya tout sur son passage, il déféqua tout. Mais l'homme n'était pas mort, il était tout à fait vivant. Il sortit. " Pourquoi t'es-tu comporté comme ça ? C'est la raison pour laquelle ce chien t'a mangé » [dit Dieu à l'homme]. « C'est pourquoi le chien t'a mangé et que maintenant il vient de te déféquer », dit-il. "Je vais partir mon père, je vais emmener [mon chien] avec moi ", dit [l'homme]. Le chien ne voulait pas. [L'homme] l'appela une nouvelle fois. Mais il ne voulait pas. Il ne voulait vraiment pas [partir avec son maître] et dit [à Dieu] : « je n'irai pas papa, vraiment pas. Avec ce qu’il m’a fait. Qu'il en emporte un autre. Il y a plein d'autres chiens ici ». Les chiens passaient leur temps à dormir. Ceux qui le veulent mangent [la nourriture] du lac. Et ceux qui veulent vont déféquer. Ceux qui veulent vont en haut ou en bas, la tête baissée [ils reniflent et cherchent avec leur truffe]. Les chiens passent leur temps à dormir. Ils avaient de la nourriture juste comme ça. Il y avait un grand lac en forme de cruche, bouillonnant et [plein] de sang à manger. Les chiens en consomment, ils en mangent un petit peu. C'est comme ça durant des années, sans que [cette nourriture] ne s'achève. [L'homme] appela un autre chien. Mais il ne voulut pas [venir avec lui]. Alors il en appela un autre. [L'ancien chien de l'homme] avait conseillé les autres chiens en leur disant «n'y allez pas, [regardez] ce qu'il m'a fait ». Il conseilla ces chiens [de ne pas suivre l'homme]. Mais il y en eut un qui dit : " allez, moi j'irai, bon j'irai ». Il s'en alla. L'homme emmena le chien avec lui. Il emmena un tout petit chien. Mais [l'ancien] chien [de l'homme] lui avait donné de bons conseils et dit : « ne va pas faire paître les animaux, ne t'acharne que sur ta nourriture, dors le jour et dors aussi la nuit ». [L'homme] le nourrissait de jour. Et le chien ne faisait que dormir. Il passait son temps à dormir de jour et à dormir aussi de nuit. Il n'aboyait pas sur le bétail et n'entendait rien [de ce qui se passait]. L'homme pleura énormément [à l'idée] de son [ancien] chien. Alors seulement, il se dit : «Bon sang, mais qu'est-ce que c'est que ce chien ? Mon autre chien n'était pas comme ça. Comme ce chien n'a pas été bien conseillé, que pourra-t-il bien faire ? Sale cleps bon sang ». L'homme se résigna et l'emmena avec lui. C'est ainsi qu'il souffrit avec ce chien car il ne pouvait rien en faire. C'est ici que s'achève ce conte.

\section{Lazaro alqumanta}

Chayqa huk ruma kasqa. Alquchansi kasqa. Mana sapallanchu runa kapun viudo. Hinaspas askha uywayuq, ashkha uhayuq, ashkha paquchayuq ima. Uhata nak'arukusqa. Chaysi alqu mikhurusqa uha aychata. Hinaspas castigaykusqa yastata alquchata. Yaqas sipisqapas " qan carajo aychata mikhuranki » nispa. Chaysi alqucha volcanta pasakapusqa. Tirra kawsachisqa. Mana wañusqachu. Volcanta pasakapusqa. Chaysi runata tapupayukun " maytan alqucha rishanman?". "Rishanmi ari alquchaqa » nispa nin. Runata tapupayukum. "Rishanmi ari alqucha » nin. Alquchanman qhipanta rishan. Chayqa alquchallansi rumahina tuta cuidantaq p'unchay cuidantaq lliwtapas. Michimuntaq hinasyá alqucha. Makillas 
p'unchaypas uywata michimun uhata paquchatapas kanispalla. Chaysi Taytanchisman chayan. Chaysi wiraqucha kashasqa chaypi. "Imamanmi hamushanki?" nispas nin. "Khayna alquchaymi karqan. Hinaspan pasakampusqa kayta ». Haqhayna qatapis alquchanta rikurukunsi. Tiramushasqa. " Haqhaytaq alquyqa » nispa nin. "Imanarankitaqri? » nispa nin. Waxan alquchata. Waxaqtinqa hamunsi alqu sutiyuq. Sutinmanta waxan Taytanchis. Chaysi wiraqucha waxaqtin hamun. Paypas alquchata sutinmanta waxan " haku ripusun " nispa. Mana munanchu hampuyta. Enteramente manapunis munanchu. Manapunis hamunchu ultimonta. Chaysi nin "nuqaqa cuidakusqayta mikhukuni arí. Tuta p'unchay cuidani tutapas makilla cuidakumi imamantapas. P'unchaypas uywatapas michikuni makilla. Chayqa cuidakusqaytan michikusqaytan nuqa mikhukuni ari. Chay uhachaykimantachu quejanki ? Imapas suwaña apanman imapas chaypas askhanpichá apamman. Nuqaqa chay aychachatachá mikhurukunipas riki. Manan riymanchu. Ripuy » nispas nin. " Mana riymanchu " nin. Taytanchispas ninsi. "Mana ripuymanchu. Castigaykuwan kaynata ruwawan 》 nispa. "Imapaqmi castigaruwanki? Lazaros kay lazaro kasqayta, imapaqmi castigaruwanki? " nispa nin. Chaysi huk alquta waxarin. Hatun alqus kasqa hatunsi kasqa. Hinaspa chaysi « come, come esto » nispas runata qhawaykachin. Alqu sayarisqa [...] Hatunsi kasqa siminpas kasqa aqnansi. Chaysi runata sayarishaqllata uquyurun alqu. Hinaspa haqhaynata phaw'arispa hisp'arparimun kaqta. Imaynata sayarishaqllatas rumata hisp'arparin. Unu Iluqsirqamun picharin picharin lliwta hisp'aykamun. Chayqa mana wañurapusqachu kawsashaqlla runaqa. Lluqsirqamum. "Imapaqmi ruwaranki chayta? Chaymantan chay alqu mikhusunki » nispa nin " chaynatan chay alqu mikhusunki, hisp'arusunki hisp'arparisunki kuman » nispa nin. Chayqa "ripusaq papay pusakapusaqchá» nin. Mana munanchu alqu. Waxan wakmanta. Mana munanchu. Manapuni enteramenteta munanchu " manapunin riymanchu papay, manapunin. Khaynatan ruwawan. Huktayá pusakuchun» nispa nin "alqukuna ashkha aswan chaypi ». Chaysi puñuyarayashallansi alqukuna. Chaysi munaq mikhukushan quchata. Munaqtaqsi hisp'aq purikushan. Munaqtaqsi wichaymanraq uraymanraq umayuq kumpasqa. Puñukushan alqukuna. Chayqa chaysi chhaynata mikhunan kasqa. Puytus hatun quchas kasqa, mikhuna yawar t'impusqas kasqa sumaq wirallaña. Chayqa llaqllarikunku imachantachá mikhunku riki alqukuna. Kaqlla watan watan mana tukukuqsi. Hinaspa huk alquta waxan. Mana munanchu. Chayqa huk alquta waxan. Consejakunsi alqukunata « ama riychischu, khaynata ruwakuwan » nispas nin. Hinaspa chay alquta consejaykurum. Chaysi " a ver nuqa risaqyá » nispas nin " bueno risaq » nispa. Chaysi rin. Chay alquchata pusakun riki. Chaysi huch'uy alquchatas pusakun. Chayqa consejaykunsi allintas chay alqu "aman michiqqa purinkichu, mikhunallatan maqanki, p'unchaypis puñuyukuy tutapis puñuyukuy » nispas nin. P'unchay mikhunata qaraykun. Chaysi lliwta puñukushan alquqa. P'unchaypas puñuyukushan tutapas puñuyukushan. Mana kanikunchu uywatapas, imatapas uyarinchu. Waqaykunsi runa alqummanta. Chayraqsi « carajo imaynataq chay alquyqa mana chaynachu huk alquyqa karan. Manas chay alquyqa allin consejasqa chayqa imatataq ruwanqari? Chayqa gart'i carajo ». Hina kapum. Runa pusakapunsiyá. Chayqa hinas mana atiqyayta sufrikapun. Chaypi tukukullantaq chay kwintupas. 